\title{
Novel SINS Initial Alignment Method under Large Misalignment Angles and Uncertain Noise Based on Nonlinear Filter
}

\author{
Bo Yang, Xiaosu Xu, Tao Zhang, Jin Sun, and Xinyu Liu \\ Key Laboratory of Micro-Inertial Instrument and Advanced Navigation Technology, Ministry of Education, \\ School of Instrument Science and Engineering, Southeast University, Nanjing 210096, China \\ Correspondence should be addressed to Xiaosu Xu; xxs@seu.edu.cn
}

Received 4 August 2016; Revised 19 December 2016; Accepted 24 January 2017; Published 19 February 2017

Academic Editor: Jean-Christophe Ponsart

Copyright (C) 2017 Bo Yang et al. This is an open access article distributed under the Creative Commons Attribution License, which permits unrestricted use, distribution, and reproduction in any medium, provided the original work is properly cited.

\begin{abstract}
For the SINS initial alignment problem under large misalignment angles and uncertain noise, two novel nonlinear filters, referred to as transformed unscented quadrature Kalman filter (TUQKF) and robust transformed unscented quadrature Kalman filter (RTUQKF), are proposed in this paper, respectively. The TUQKF sets new deterministic sigma points to address the nonlocal sampling problem and improve the numerical accuracy. The RTUQKF is the combination of $H_{\infty}$ technique and TUQKF. It improves the accuracy and robustness of state estimation. Simulation results indicate that TUQKF performs better than traditional filters when misalignment angles are large. Turntable and vehicle experiments results indicate that, under the condition of uncertain noise, the performances of RTUQKF are better than other filters and more robust. These two methods can effectively further increase precision and convergence speed of SINS initial alignment.
\end{abstract}

\section{Introduction}

Initial alignment is one of the critical and difficult problems for inertial navigation system (INS). The essential purpose of initial alignment for strapdown INS (SINS) is to determine the attitude matrix between body frame and navigation frame $[1,2]$. The Kalman filter (KF) is the mostly used technique to solve the problem of initial alignment. However, it can only deal with initial alignment under small misalignment angles [3]. Large misalignment angles and uncertain noise are two main problems existing in initial alignment in different application environments $[4,5]$. The nonlinear model of SINS and nonlinear methods are developed to solve the alignment problem. In [6], a modeling method of nonlinear model of SINS was proposed. The widely used nonlinear filtering method in engineering is extended KF (EKF). The principle of EKF is simple and it has high computationally efficient [7]. However, the performance of EKF would decrease if the system has strong nonlinear characters [8]. The unscented KF (UKF) and cubature KF (CKF) were developed to overcome this problem $[9,10]$. These two algorithms were applied to
SINS initial alignment and got better effects than EKF [11, 12]. Although the derivation method of CKF is different from standard UKF, it is virtually a special case of the UKF [13]. It has pointed out that CKF has better precisions than UKF for high-dimensional problem [10]. However, it has proved that CKF suffers from nonlocal sampling problem which will lead to estimation errors in high-dimensional and strong nonlinear situations [14]. From [6] and Section 5, it is shown that nonlinear model of SINS includes a lot of trigonometric operations and the state dimensions are more than 10 , so the SINS initial alignment problem under large misalignment angles is the high-dimensional problem coupled with strong nonlinear model. Therefore, in [14], new set of sigma points was designed to solve this problem and the novel algorithm is known as transformed unscented KF (TUKF). In this paper, in order to further increase accuracy of SINS initial alignment, the transformed unscented quadrature KF (TUQKF) was proposed based on TUKF. TUQKF is an extended version of TUKF. It is proved that, under single Gauss-Laguerre quadrature rule, TUQKF degenerates to TUKF and TUQKF 
exhibits better numerical characteristics than TUKF when using high-order Gauss-Laguerre rule.

For the other problem, SINS initial alignment with uncertain noise, if environment noise is not Gaussian white noise, the traditional nonlinear filtering method mentioned above will produce a greater estimation error. One solution for this problem is to use the $H_{\infty}$ technique to improve the robustness of filter. The $H_{\infty}$ filter is more robust and has better precision than standard KF under uncertain noise $[15,16]$. However, standard $H_{\infty}$ filter can only fulfill the linear filtering problems. In the recent two decades, $H_{\infty}$ filter was extended to nonlinear problems by combing with nonlinear filters. In [17], the $H_{\infty}$ filter was combined with EKF and the new algorithm known as robust extended KF (REKF) was proposed. In this framework, the $H_{\infty}$ filter was combined with UKF and CKF. These two new algorithms are known as robust unscented KF (REKF) and cubature $H_{\infty}$ filter $\left(\mathrm{CH}_{\infty} \mathrm{F}\right)[16,18]$. In [18], the RUKF was applied to the SINS initial alignment with colored noise and achieved better accuracy than UKF. In this paper, in order to further increase the performance of filter for SINS initial alignment, $H_{\infty}$ filter has been combined with TUQKF and a novel algorithm named as robust transformed unscented quadrature KF (RTUQKF) was proposed. The proposed TUQKF and RTUQKF were used for SINS initial alignment, respectively, and compared with the square-root cubature KF (SRCKF) in mathematical simulation, turntable experiment, and vehicle experiment.

The rest of paper is organized as follows. Section 2 reviews the conventional nonlinear filters. Section 3 derives the TUQKF algorithm. The RTUQKF algorithm is derived in Section 4. The nonlinear model of SINS is established in Section 5. Three experiments are conducted in Section 6 to verify the effectiveness of TUQKF and RTUQKF. Finally, Conclusions are drawn in Section 7.

\section{Conventional Nonlinear Filters}

The general discrete nonlinear model is given by

$$
\begin{aligned}
& \mathbf{x}_{k}=f\left(\mathbf{x}_{k-1}\right)+\boldsymbol{\omega}_{k-1} \\
& \mathbf{z}_{k}=h\left(\mathbf{x}_{k}\right)+\mathbf{v}_{k},
\end{aligned}
$$

where $\mathbf{x}_{k} \in R^{n}$ and $\mathbf{z}_{k} \in R^{m}$ are state and measurement, $f(\cdot)$ is nonlinear state function, and $h(\cdot)$ is nonlinear measurement function. $\boldsymbol{\omega}_{k-1}$ and $\mathbf{v}_{k}$ are uncorrelated zero-mean white Gaussian noise with variances $\mathbf{Q}_{k}$ and $\mathbf{R}_{k}$, respectively.

The state vector $\mathbf{x}_{k}$ can be estimated by general Gaussian approximation filter. The heart of the Gaussian approximation filter is to evaluate the integral as follows [19]:

$$
I(f)=\int_{R^{n}} F(\mathbf{x}) \times N(\mathbf{x} ; \widehat{\mathbf{x}}, \mathbf{P}) d \mathbf{x}_{k},
$$

where $F(\mathbf{x})$ is an arbitrary nonlinear function.
Gauss-Hermite quadrature rule [20], unscented transformation method [8], and the cubature rule [10] are generally used to approximate the above integral:

$$
\begin{array}{rl}
\int_{R^{n}} & F(\mathbf{x}) \times N(\mathbf{x} ; \widehat{\mathbf{x}}, \mathbf{P}) d \mathbf{x} \\
= & \int_{R^{n}} F(\mathbf{S} \mathbf{x}+\widehat{\mathbf{x}}) \times N(\mathbf{x} ; 0, \mathbf{I}) d \mathbf{x} \\
& \approx \sum_{i=1}^{N_{s}} W_{i} F\left(\mathbf{S} \boldsymbol{\beta}_{i}+\widehat{\mathbf{x}}\right),
\end{array}
$$

where $\boldsymbol{\beta}_{i}$ is quadrature points, $W_{i}$ is corresponding weights, and $\mathbf{S}$ is square root of $\mathbf{P}$; namely, $\mathbf{P}=\mathbf{S S}^{T}$.

Therefore, the main purpose of evaluating integral (2) is to obtain quadrature points $\boldsymbol{\beta}_{i}$ and weights $W_{i}$.

Using Gauss-Hermite quadrature rule to approximate the integral (2), the total number of points increases exponentially with the dimension $n$ and it leads to the curse of dimensionality problem [21]. So, it is not suitable for SINS initial alignment which is the high-dimensional problem. The unscented transformation method and cubature rule also bear the problem of accuracy decline for high-dimensional or strong nonlinear problems $[10,14]$. To solve these problems, the TUKF selects novel sigma points to approximate the integral (2); the $\boldsymbol{\beta}_{i}$ and $W_{i}$ are given by [14]

$$
\begin{aligned}
\boldsymbol{Y}_{i} & =\left(\gamma_{i, 1}, \gamma_{i, 2}, \ldots, \gamma_{i, n}\right)^{T} ; \quad i=1,2, \ldots, 2 n, \\
\boldsymbol{\beta}_{i} & =\sqrt{n} \Upsilon_{i} ; \quad i=1,2, \ldots, 2 n, \\
W_{i} & =\frac{1}{2 n} ; \quad i=1,2, \ldots, 2 n,
\end{aligned}
$$

where

$$
\begin{aligned}
\gamma_{i, 2 r-1} & =\sqrt{\frac{2}{n}} \cos \frac{(2 r-1) i \pi}{n} \\
\gamma_{i, 2 r} & =\sqrt{\frac{2}{n}} \sin \frac{(2 r-1) i \pi}{n},
\end{aligned}
$$

where $i=1,2, \ldots, 2 n, r=1,2, \ldots,[n / 2]$, and $[n / 2]$ denotes the greatest integer less than $n / 2 . r_{i, n}=(-1)^{i-1}$ when $n$ is odd.

In this paper, we propose a new sigma point selection strategy based on cubature rule and TUKF to further improve the performance of filter for high-dimensional problem.

\section{Transformed Unscented Quadrature Kalman Filter}

3.1. Cubature Rule and Transformed Cubature Points. Putting Gaussian probability density function into (2) yields

$$
I(f)=\frac{1}{\sqrt{\mathbf{P}(2 \pi)^{n}}} \int_{R^{n}} f(\mathbf{x}) e^{-(\mathbf{x}-\boldsymbol{\mu})(\mathbf{x}-\mu)^{T} / 2 \mathbf{P}} d \mathbf{x},
$$

where $\boldsymbol{\mu}$ and $\mathbf{P}$ are the mean and variance of state vector $\mathbf{x}$, respectively. 
Equation (6) can be transformed by spherical-radial cubature rule $[10,22]$ :

$$
\begin{aligned}
& I(f) \\
& \quad=\frac{1}{\sqrt{(2 \pi)^{n}}} \int_{r=0}^{\infty} \int_{U_{n}}[f(\mathbf{S} r \mathbf{Z}+\boldsymbol{\mu}) d s(\mathbf{Z})] r^{n-1} e^{-r^{2} / 2} d r,
\end{aligned}
$$

where $\mathbf{x}=\mathbf{S} r \mathbf{Z}+\boldsymbol{\mu}$, $\mathbf{S}$ denotes Cholesky decomposition of $\mathbf{P}$, namely $\mathbf{P}=\mathbf{S S}^{T},\|\mathbf{Z}\|=1$, and $U_{n}$ denotes surface of unit hypersphere.

First, compute integral

$$
\int_{U_{n}}[f(\mathbf{S} r Z+\boldsymbol{\mu}) d s(\mathbf{Z})]
$$

Integral (8) can be approximated by [10]

$$
\int_{U_{n}}[f(\mathbf{S} r Z+\boldsymbol{\mu}) d s(\mathbf{Z})] \approx\left(\sum_{i=1}^{k} f\left(\mathbf{S} r \boldsymbol{\alpha}_{i}+\boldsymbol{\mu}\right)\right) w,
$$

where $\boldsymbol{\alpha}_{i}$ are space points and $\left\|\boldsymbol{\alpha}_{i}\right\|=1, k$ denotes number of space points, and $w$ denotes corresponding weights.

It can be seen that the computational precision of integral (7) depends on $\boldsymbol{\alpha}_{i}$ and $w$. Performance of filters is also influenced by $\boldsymbol{\alpha}_{i}$ and $w$.

The $\boldsymbol{\alpha}_{i}$ can be selected by

$$
\boldsymbol{\alpha}_{i}=\left(\alpha_{i, 1}, \alpha_{i, 2}, \ldots, \alpha_{i, n}\right)^{T},
$$

where

$$
\begin{aligned}
\alpha_{i, 2 r-1} & =\sqrt{\frac{2}{n}} \cos \frac{(2 r-1) i \pi}{n}, \\
\alpha_{i, 2 r} & =\sqrt{\frac{2}{n}} \sin \frac{(2 r-1) i \pi}{n},
\end{aligned}
$$

where $i=1,2, \ldots, 2 n$ and $r=1,2, \ldots,[n / 2]$. If $n$ is odd, $r_{i, n}=$ $(-1)^{i-1}$.

In [14], it has been proved that $\boldsymbol{\alpha}=\left[\boldsymbol{\alpha}_{1}, \boldsymbol{\alpha}_{2}, \ldots, \boldsymbol{\alpha}_{2 n}\right]$ is an orthogonal matrix, so $\left\|\boldsymbol{\alpha}_{i}\right\|=1$.

The $w$ can be calculated by [10]

$$
w=\frac{2 \sqrt{\pi^{n}}}{2 n \Gamma(n / 2)} .
$$

3.2. Gauss-Laguerre Quadrature Rule. Bringing (9) and (12) into (6) and letting $\lambda=r^{2} / 2$ result in

$$
\begin{aligned}
& I(f) \\
& \quad \approx \frac{1}{2 n \Gamma(n / 2)} \int_{\lambda=0}^{\infty} \sum_{i=1}^{2 n} f\left(\mathbf{s} \sqrt{2 \lambda} \boldsymbol{\alpha}_{i}+\boldsymbol{\mu}\right) \lambda^{(n / 2-1)} e^{-\lambda} d \lambda .
\end{aligned}
$$

Define $g(\lambda)$ as

$$
g(\lambda)=\sum_{i=1}^{2 n} f\left(\mathbf{s} \sqrt{2 \lambda} \boldsymbol{\alpha}_{i}+\boldsymbol{\mu}\right) .
$$

Computing integral (6) is transformed into computing integral (13). First, calculate the integral

$$
\int_{\lambda=0}^{\infty} g(\lambda) \lambda^{(n / 2-1)} e^{-\lambda} d \lambda .
$$

Gauss-Laguerre quadrature rule can be used to approximate integral (15) [10, 22]:

$$
\int_{x=0}^{\infty} g(\mathbf{x}) \mathbf{x}^{\gamma} e^{-x} d \mathbf{x} \approx \sum_{i=1}^{m} A_{i} g\left(\lambda_{i}\right),
$$

where $\lambda_{i}$ is quadrature point and $A_{i}$ is corresponding weight.

$\lambda_{i}$ can be calculated from

$$
L_{m}^{\gamma}(\lambda)=(-1)^{m} \lambda^{-\gamma} e^{\lambda} \frac{d^{m}}{d \lambda^{m}} \lambda^{\gamma+m} e^{-\lambda}=0 .
$$

The weights can be determined as

$$
A_{i}=\frac{m ! \Gamma(\gamma+m+1)}{\lambda_{i}\left[\dot{L}_{m}^{\gamma}(\lambda)\right]^{2}} .
$$

The precision of the integral depends on the number of quadrature points. The higher the number of quadrature points is the better the precision would be [22]. In addition, the quadrature points and weights of Gauss-Laguerre quadrature are independent of nonlinear function $f(\mathbf{x})$, so quadrature points and weights always exist and can be computed and stored offline. yields

Bringing approximate results of integral (15) into (13)

$$
I(f) \approx \sum_{j=1}^{m} \sum_{i=1}^{2 n} f\left(\mathbf{s} \boldsymbol{\xi}_{j}+\boldsymbol{\mu}\right) W_{j},
$$

where

$$
\begin{aligned}
\boldsymbol{\xi}_{j} & =\sqrt{2 \lambda_{j}} \boldsymbol{\alpha}_{i}, \\
W_{j} & =\frac{A_{j}}{2 n \Gamma(n / 2)},
\end{aligned}
$$

where $\boldsymbol{\xi}_{j}$ is sigma points and $W_{j}$ is corresponding weight of TUQKF.

If integral (15) is solved with $m$ order Gauss-Laguerre quadrature rule, the TUQKF is named as $m$-order TUQKF (TUQKF- $m$ ).

3.3. Analyses of TUQKF. If using first-order Gauss-Laguerre quadrature rule, quadrature point and weight are $\lambda_{1}=\sqrt{n / 2}$ and $A_{1}=\Gamma(n / 2)$. Putting $\lambda_{1}$ and $A_{1}$ into (20) yields

$$
\begin{aligned}
\boldsymbol{\xi}_{i} & =\sqrt{n} \boldsymbol{\alpha}_{i} ; \quad i=1,2, \ldots, 2 n, \\
W_{i} & =\frac{1}{2 n} ; \quad i=1,2, \ldots, 2 n .
\end{aligned}
$$

Comparing (4) and (21), it can be seen that the sigma points and weights of the TUKF and the TUQKF-1 are the 
same. This means TUQKF-1 coincides with TUKF. So, TUKF is also derived in this paper from another point of view and TUQKF is an extended version of the TUKF. The precision of the filter is influenced by the order of Gauss-Laguerre rule. The higher the number of quadrature points is the higher the calculation precision of integral (2) would be. The higher the calculation accuracy of integral (2) the better the accuracy of the filter would be. So, mathematically, the performance of the TUQKF- $m$ is better than the TUKF when $m>1$.

Compared to EKF, TUQKF does not need to calculate Jacobian matrices and is more suitable for the system which has strong characters of nonlinear. Compared to UKF, TUQKF maintains the positive definiteness of the weight and the covariance matrix for high-dimensional problem. Compared to CKF, TUQKF also solves the nonlocal sampling problem. So, TUQKF is suitable for high-dimensional problem coupled with strong nonlinear model.

Finally, discuss the computational efficiency. TUQKF- $m$ requires $M=2 \mathrm{~nm}$ sigma points, so it is computationally more efficient than the Gauss-Hermite filter which requires $m^{n}$ sigma points. However, the computational cost of TUQKF- $m$ is slightly higher than CKF and TUKF when $m>1$.

When using the TUQKF- $m$, the balance between the accuracy and the computational cost should be considered. If the dimension of state vector is high, $m$ should not be chosen very large. In addition, in practice, the assumption that the posterior density functions obey the Gauss distribution is hardly met. So, a large value of $m$ would cause the problems such as "over fitting." Considering the above problems, $m$ was set to 2 in this paper.

\subsection{TUQKF Algorithm Flow}

\subsubsection{Initial Filter}

(i) Initial $\widehat{\mathbf{x}}_{0}$ and $\mathbf{P}_{0}$ by

$$
\begin{aligned}
\widehat{\mathbf{x}}_{0} & =E\left(\mathbf{x}_{0}\right), \\
\mathbf{P}_{0} & =E\left(\left(\widehat{\mathbf{x}}_{0}-\mathbf{x}_{0}\right)\left(\widehat{\mathbf{x}}_{0}-\mathbf{x}_{0}\right)^{T}\right) .
\end{aligned}
$$

(ii) Compute sigma point $\xi_{i}$ and weight $W_{i}(i=1,2, \ldots$, $M)$.

\subsubsection{Time Update}

(i) Factorize

$$
\mathbf{P}_{k-1 \mid k-1}=\mathbf{S}_{k-1 \mid k-1} \mathbf{S}_{k-1 \mid k-1}^{T} .
$$

(ii) Evaluate and update transformed unscented quadrature points

$$
\begin{aligned}
& \mathbf{X}_{i, k-1 \mid k-1}=\mathbf{S}_{k-1 \mid k-1} \boldsymbol{\xi}_{i}+\widehat{\mathbf{x}}_{k-1 \mid k-1} \quad i=1,2, \ldots, M, \\
& \mathbf{X}_{i, k-1 \mid k-1}^{*}=f\left(\mathbf{X}_{i, k-1 \mid k-1}\right) \quad i=1,2, \ldots, M .
\end{aligned}
$$

(iii) Estimate predicted state and error covariance

$$
\begin{aligned}
\widehat{\mathbf{x}}_{k \mid k-1}= & \sum_{i=1}^{M} W_{i} \mathbf{X}_{i, k-1 \mid k-1}^{*}, \\
\mathbf{P}_{k \mid k-1}= & \sum_{i=1}^{M} W_{i} \mathbf{X}_{i, k-1 \mid k-1}^{*} \mathbf{X}_{i, k-1 \mid k-1}^{* T}-\widehat{\mathbf{x}}_{k \mid k-1} \widehat{\mathbf{x}}_{k \mid k-1}^{T} \\
& +\mathbf{Q}_{k-1} .
\end{aligned}
$$

\subsubsection{Measurement Update}

(i) Factorize

$$
\mathbf{P}_{k \mid k-1}=\mathbf{S}_{k \mid k-1} \mathbf{S}_{k \mid k-1}^{T} .
$$

(ii) Evaluate and update transformed unscented quadrature points

$$
\begin{aligned}
& \mathbf{X}_{i, k \mid k-1}=\mathbf{S}_{k \mid k-1} \boldsymbol{\xi}_{i}+\widehat{\mathbf{x}}_{k-1 \mid k-1} \quad i=1,2, \ldots, M, \\
& \mathbf{Z}_{i, k \mid k-1}=h\left(\mathbf{X}_{i, k-1 \mid k-1}\right) \quad i=1,2, \ldots, M .
\end{aligned}
$$

(iii) Estimate predicted measurement

$$
\widehat{\mathbf{z}}_{k \mid k-1}=\sum_{i=1}^{M} W_{i} \mathbf{Z}_{i, k-1 \mid k-1} .
$$

(iv) Estimate innovation covariance and cross-covariance matrix

$$
\begin{aligned}
& \mathbf{P}_{z z, k \mid k-1}=\sum_{i=1}^{M} W_{i} \mathbf{Z}_{i, k-1 \mid k-1} \mathbf{z}_{i, k-1 \mid k-1}^{T}-\widehat{\mathbf{z}}_{k \mid k-1} \widehat{\mathbf{z}}_{k \mid k-1}^{T}, \\
& \mathbf{P}_{x z, k \mid k-1}=\sum_{i=1}^{M} W_{i} \mathbf{X}_{i, k-1 \mid k-1} \mathbf{Z}_{i, k-1 \mid k-1}^{T}-\widehat{\mathbf{x}}_{k \mid k-1} \widehat{\mathbf{z}}_{k \mid k-1}^{T} .
\end{aligned}
$$

(v) Estimate the gain

$$
\mathbf{K}=\mathbf{P}_{x z, k \mid k-1}\left(\mathbf{P}_{z z, k \mid k-1}+\mathbf{R}_{k}\right)^{-1} .
$$

(vi) Estimate updated state and corresponding error covariance

$$
\begin{aligned}
& \widehat{\mathbf{x}}_{k \mid k}=\widehat{\mathbf{x}}_{k \mid k-1}+\mathbf{K}\left(\mathbf{z}_{k}-\widehat{\mathbf{z}}_{k \mid k-1}\right), \\
& \mathbf{P}_{k \mid k}=\mathbf{P}_{k \mid k-1}-\mathbf{K}\left(\mathbf{P}_{z z, k \mid k-1}+\mathbf{R}_{k}\right) \mathbf{K}^{T} .
\end{aligned}
$$

Measurement function described in (1) can be simplified as the following linear function:

$$
\mathbf{z}_{k}=\mathbf{H}_{k} \mathbf{x}_{k}+\mathbf{v}_{k}
$$

where $\mathbf{H}_{k}$ is the measurement matrix.

Measurement-update steps (26) (29) can be simplified as

$$
\begin{aligned}
\widehat{\mathbf{z}}_{k \mid k-1} & =\mathbf{H}_{k} \widehat{\mathbf{x}}_{k \mid k-1}, \\
\mathbf{P}_{z z, k \mid k-1} & =\mathbf{H}_{k} \mathbf{P}_{k \mid k-1} \mathbf{H}_{k}^{T}, \\
\mathbf{P}_{x z, k \mid k-1} & =\mathbf{P}_{k \mid k-1} \mathbf{H}_{k}^{T} .
\end{aligned}
$$




\section{Robust Transformed Unscented Quadrature Kalman Filter}

The discrete nonlinear model can be expressed as

$$
\begin{aligned}
& \mathbf{x}_{k}=f\left(\mathbf{x}_{k-1}\right)+\boldsymbol{\omega}_{k-1}, \\
& \mathbf{z}_{k}=h\left(\mathbf{x}_{k}\right)+\mathbf{v}_{k}, \\
& \mathbf{y}_{k}=\mathbf{L}_{k} \mathbf{x}_{k},
\end{aligned}
$$

where $\mathbf{x}_{k} \in R^{n}$ and $\mathbf{z}_{k} \in R^{m}$ are the state and measurement. $f(\cdot)$ is nonlinear state function, and $h(\cdot)$ is nonlinear measurement function. $\boldsymbol{\omega}_{k-1}$ and $\mathbf{v}_{k}$ are noise with uncertain statistical properties. $\mathbf{y}_{k} \in R^{n}$ is the signal to be estimated and $\mathbf{L}_{k}$ is known.

Nonlinear filters discussed above are derived under the condition of zero-mean Gaussian white noise. However, statistical properties of $\boldsymbol{\omega}_{k-1}$ and $\mathbf{v}_{k}$ are uncertain in (37). We can assume that the noise is zero-mean Gaussian white noise with variances $\mathbf{Q}$ and $\mathbf{R}$. However, if the assumption is false, the nonlinear filters discussed above will produce greater estimation errors. Combining nonlinear filters with $H_{\infty}$ technique can improve the performance of filters under the different noise with different statistical properties.

4.1. REKF Algorithm. The robust extended Kalman filter (REKF) is the combination of $H_{\infty}$ filter and EKF [17]. It contains the following two steps:

Time update:

$$
\begin{aligned}
& \widehat{\mathbf{x}}_{k \mid k-1}=f\left(\widehat{\mathbf{x}}_{k-1 \mid k-1}\right), \\
& \mathbf{P}_{k \mid k-1}=\mathbf{F}_{k-1} \mathbf{P}_{k \mid k-1} \mathbf{F}_{k-1}^{T}+\mathbf{Q}_{k-1},
\end{aligned}
$$

where $\mathbf{F}_{k-1}$ is Jacobian matrix of $f(\cdot)$ computed at $\widehat{\mathbf{x}}_{k-1 \mid k-1}$.

Measurement update:

$$
\begin{aligned}
\widehat{\mathbf{x}}_{k \mid k}= & \widehat{\mathbf{x}}_{k \mid k-1}+\mathbf{K}\left[\mathbf{z}_{k}-h\left(\widehat{\mathbf{x}}_{k \mid k-1}\right)\right] \\
\mathbf{K}= & \mathbf{P}_{k \mid k-1} \mathbf{H}_{k}^{T}\left(\mathbf{H}_{k} \mathbf{P}_{k \mid k-1} \mathbf{H}_{k}^{T}+\mathbf{R}_{k}\right)^{-1}, \\
\mathbf{P}_{k \mid k}= & \mathbf{P}_{k \mid k-1} \\
& -\mathbf{P}_{k \mid k-1}\left[\begin{array}{ll}
\mathbf{H}_{k}^{T} & \mathbf{L}_{k}^{T}
\end{array}\right] \mathbf{R}_{e, k}^{-1}\left[\begin{array}{ll}
\mathbf{H}_{k}^{T} & \mathbf{L}_{k}^{T}
\end{array}\right]^{T} \mathbf{P}_{k \mid k-1}, \\
\mathbf{R}_{e, k}= & {\left[\begin{array}{cc}
\mathbf{R} & \mathbf{0} \\
\mathbf{0} & -\gamma^{2} \mathbf{I}
\end{array}\right]+\left[\begin{array}{ll}
\mathbf{H}_{k}^{T} & \mathbf{L}_{k}^{T}
\end{array}\right]^{T} \mathbf{P}_{k \mid k-1}\left[\begin{array}{ll}
\mathbf{H}_{k}^{T} & \mathbf{L}_{k}^{T}
\end{array}\right], }
\end{aligned}
$$

where $\mathbf{H}_{k}$ is Jacobian matrix of $h(\cdot)$ computed at $\widehat{\mathbf{x}}_{k \mid k-1} \cdot \gamma$ is the bound which can be chosen by designer.

If, and only if, the condition is satisfied,

$$
\mathbf{P}_{k \mid k}-\gamma^{-2} \mathbf{L}_{k}^{T} \mathbf{L}_{k}>0 \quad 0 \leq k \leq N,
$$

the filter can be designed to achieve the desired accuracy by iterating $\gamma$ to arrive at a suboptimal solution $[23,24]$.

It can be easily shown that REKF reverts to EKF with $\gamma \rightarrow \infty$. Thus, $\gamma$ can be thought as a tuning parameter to control the trade-off between performance of $H_{\infty}$ and minimum variance performance $[17,25]$. Equation (43) will be satisfied easily with a large value of $\gamma$, but the large value of $\gamma$ reduces the robustness of the filter. If we set $\gamma$ to a small value, there is no guarantee that (43) will be satisfied during the process of filtering. So, the selection of the parameter $\gamma$ is very important. One method to choose parameter $\gamma$ will be given in the next part.

4.2. Robust Transformed Unscented Quadrature Kalman Filter. In this part, the RTUQKF algorithm will be derived based on REKF framework.

Equations (38) in timing update of REKF can be replaced by the (23) (25) in timing update of TUQKF and the Jacobian matrix $\mathbf{H}_{k}$ can be replaced by the method based on the following two approximations [26]:

$$
\begin{aligned}
& \mathbf{P}_{z z, k \mid k-1} \approx \mathbf{H}_{k} \mathbf{P}_{k \mid k-1} \mathbf{H}_{k}^{T}, \\
& \mathbf{P}_{x z, k \mid k-1} \approx \mathbf{P}_{k \mid k-1} \mathbf{H}_{k}^{T} .
\end{aligned}
$$

For SINS initial alignment, the signal which needs to be estimated is state $\mathbf{x}_{k}$, so $\mathbf{L}_{k}$ can be set to I. Putting $\mathbf{L}_{k}=\mathbf{I}$, (44) into (41) (42), results in

$$
\begin{aligned}
& \mathbf{P}_{k \mid k}=\mathbf{P}_{k \mid k-1}-\left[\begin{array}{ll}
\mathbf{P}_{k \mid k-1} \mathbf{H}_{k}^{T} & \mathbf{P}_{k \mid k-1}
\end{array}\right] \mathbf{R}_{e, k}^{-1}\left[\begin{array}{c}
\mathbf{H}_{k} \mathbf{P}_{k \mid k-1} \\
\mathbf{P}_{k \mid k-1}
\end{array}\right] \\
& \approx \mathbf{P}_{k \mid k-1}-\left[\begin{array}{ll}
\mathbf{P}_{x z, k \mid k-1} & \mathbf{P}_{k \mid k-1}
\end{array}\right] \mathbf{R}_{e, k}^{-1}\left[\begin{array}{c}
\mathbf{P}_{x z, k \mid k-1}^{T} \\
\mathbf{P}_{k \mid k-1}
\end{array}\right] \\
& \mathbf{R}_{e, k}=\left[\begin{array}{cc}
\mathbf{R} & \mathbf{0} \\
\mathbf{0} & -\gamma^{2} \mathbf{I}
\end{array}\right]+\left[\begin{array}{cc}
\mathbf{H}_{k} \mathbf{P}_{k \mid k-1} \mathbf{H}_{k}^{T} & \mathbf{H}_{k} \mathbf{P}_{k \mid k-1} \\
\mathbf{P}_{k \mid k-1} \mathbf{H}_{k}^{T} & \mathbf{P}_{k \mid k-1}
\end{array}\right] \\
& \approx\left[\begin{array}{cc}
\mathbf{R}+\mathbf{P}_{z z, k \mid k-1} & \mathbf{P}_{x z, k \mid k-1}^{T} \\
\mathbf{P}_{x z, k \mid k-1} & -\gamma^{2} \mathbf{I}+\mathbf{P}_{k \mid k-1}
\end{array}\right] .
\end{aligned}
$$

$\mathbf{P}_{z z, k \mid k-1}$ and $\mathbf{P}_{x z, k \mid k-1}$ can be calculated by (29) or (35) (36). The RTUQKF algorithm can be summarized as follows:

(1) Initial $\widehat{\mathbf{x}}_{0}$ and $\mathbf{P}_{0}$ by (22).

(2) Time update: compute (23) (25).

(3) Measurement update: compute (26) (31) and (45).

If measurement function described in (37) can be simplified as linear function (33), the measurement-update steps (26) (29) can be simplified as (34) (36).

If $H_{\infty}$ filter is combined with TUQKF- $m$, the proposed RTUQKF is named as $m$-order RTUQKF (RTUQKF- $m$ ).

One way to choose parameter $\gamma$ was proposed in [27].

Applying the matrix inversion lemma for (41) and $\mathbf{P}_{k \mid k}$ should maintain the positive definiteness during the process of filtering, so it can be easily shown that

$$
\mathbf{P}_{k \mid k}^{-1}=\mathbf{P}_{k \mid k-1}^{-1}+\mathbf{H}_{k}^{T} \mathbf{R}^{-1} \mathbf{H}_{k}-\gamma^{2} \mathbf{I}>\mathbf{0} .
$$

Thus, $\gamma$ can be selected as

$$
\gamma^{2}=\alpha \max \left\{\operatorname{eig}\left(\mathbf{P}_{k \mid k-1}^{-1}+\mathbf{H}_{k}^{T} \mathbf{R}^{-1} \mathbf{H}_{k}\right)^{-1}\right\},
$$


where $\max \left\{\operatorname{eig}(A)^{-1}\right\}$ denotes the maximum eigenvalue of the matrix $A^{-1}$ and $\alpha$ is a scalar larger than one.

Putting (44) into (47), $\gamma$ in RTUQKF can be selected as

$$
\begin{aligned}
\gamma^{2} & =\alpha \max \left\{\operatorname { e i g } \left(\mathbf{P}_{k \mid k-1}^{-1}\right.\right. \\
& \left.\left.+\mathbf{P}_{k \mid k-1}^{-1} \mathbf{P}_{x z, k \mid k-1} \mathbf{R}^{-1}\left[\mathbf{P}_{k \mid k-1}^{-1} \mathbf{P}_{x z, k \mid k-1}\right]^{T}\right)^{-1}\right\} .
\end{aligned}
$$

4.3. A Comparison of TUQKF with RTUQKF. Comparing RTUQKF and TUQKF, it can be found that their essential distinction is that $\mathbf{P}_{k \mid k}$ is different. The parameter $\gamma$ is added in the RTUQKF and $\mathbf{K}$ is adjusted by changing the value of $\mathbf{P}_{k \mid k}$. So, RTUQKF improves the robustness of system by the parameter $\gamma$. Similarly, the RTUQKF reverts to TUKF with $\gamma \rightarrow \infty$.

TUQKF can also be used under the condition of uncertain noise, although its performance may not be better than RTUQKF. However, RTUQKF is more complex than TUQKF and its computational cost is also slightly higher than TUQKF. In particular, a false parameter $\gamma$ would lead to filter divergence. So, in this paper, these two new methods were proposed and in practice, one of them can be selected according to demand and actual situation.

\section{Nonlinear Model of SINS}

The auxiliary external information such as velocity reference is necessary when using nonlinear filters to solve the problem of SINS initial alignment. The velocity reference can be given by GPS in most case. If the navigation system is underwater, the velocity reference can be given by DVL. In this paper, the nonlinear model of SINS is established based on [6].

5.1. Subsection Establishment of the State Equation. In the model of SINS, the navigation frame ( $n$ frame) is usually chosen as the geographic coordinate system (ENU) and the
SINS calculation platform coordinate can be described as $n^{\prime}$ frame. The body frame ( $b$ frame) is fixed at the center of body, the right direction is defined as $x$-axis, and the front and the upward directions are defined as $y$-axis and $z$ axis, respectively. Three Euler angles, yaw, pitch, and roll, are denoted as $\psi, \theta$, and $\gamma$, respectively. Misalignment angles can be denoted by $\phi_{u}, \phi_{e}$, and $\phi_{n}$. $\mathbf{C}_{b}^{n}$ is the rotation transformation matrix from $n$ frame to $b$ frame. The true attitude angles can be denoted as $\boldsymbol{\varphi}=\left[\begin{array}{lll}\theta & \gamma & \psi\end{array}\right]^{T}$. The true velocity information can be denoted as $\mathbf{v}_{s}^{n}=\left[\begin{array}{lll}v_{e}^{n} & v_{n}^{n} & v_{u}^{n}\end{array}\right]^{T}$. The attitude angles calculated by SINS can be denoted as $\widetilde{\boldsymbol{\varphi}}=\left[\begin{array}{lll}\widetilde{\theta} & \widetilde{\gamma} & \widetilde{\psi}\end{array}\right]^{T}$. The velocity information calculated by SINS can be denoted as $\widetilde{\mathbf{v}}_{s}^{n}=\left[\begin{array}{lll}\widetilde{v}_{e}^{n} & \widetilde{v}_{n}^{n} & \widetilde{v}_{u}^{n}\end{array}\right]^{T}$. Denote the vector $\boldsymbol{\phi}=\boldsymbol{\varphi}-\widetilde{\boldsymbol{\varphi}}=$ $\left[\begin{array}{lll}\phi_{u} & \phi_{e} & \phi_{n}\end{array}\right]^{T}$. The velocity error can be denoted as $\delta \mathbf{v}^{n}=\mathbf{v}_{s}^{n}-$ $\widetilde{\mathbf{v}}_{s}^{n}=\left[\begin{array}{lll}\delta v_{e}^{n} & \delta v_{n}^{n} & \delta v_{u}^{n}\end{array}\right]^{T}$. The nonlinear error equations can be described by

$$
\begin{aligned}
\dot{\boldsymbol{\phi}}= & \mathbf{C}_{\omega}^{-1}\left[\left(I-\mathbf{C}_{n}^{n^{\prime}}\right) \widetilde{\boldsymbol{\omega}}_{i n}^{n}+\mathbf{C}_{n}^{n^{\prime}} \delta \boldsymbol{\omega}_{i n}^{b}-\mathbf{C}_{b}^{n^{\prime}}\left(\boldsymbol{\varepsilon}^{b}+\mathbf{w}_{g}^{b}\right)\right], \\
\delta \dot{\mathbf{v}}^{n} & \\
= & {\left[\left(\mathbf{I}-\mathbf{C}_{n^{\prime}}^{n}\right)\right] \mathbf{C}_{b}^{n^{\prime}} \widetilde{\mathbf{f}}^{b}-\left(2 \delta \boldsymbol{\omega}_{i e}^{n}+\delta \boldsymbol{\omega}_{e n}^{n}\right) \times \widetilde{\mathbf{v}}^{n} } \\
& \quad-\left(2 \widetilde{\boldsymbol{\omega}}_{i e}^{n}+\widetilde{\boldsymbol{\omega}}_{e n}^{n}\right) \times \delta \mathbf{v}^{n}+\mathbf{C}_{n^{\prime}}^{n} \mathbf{C}_{b}^{n^{\prime}}\left(\nabla^{b}+\mathbf{w}_{a}^{b}\right),
\end{aligned}
$$

where $\boldsymbol{\varepsilon}^{b}=\left[\begin{array}{lll}\varepsilon_{x}^{b} & \varepsilon_{y}^{b} & \varepsilon_{z}^{b}\end{array}\right]$ and $\mathbf{w}_{g}^{b}$ are the gyroscope constant errors and gyroscope random errors in $b$ frame. $\nabla^{b}=$ $\left[\begin{array}{lll}\nabla_{x}^{b} & \nabla_{y}^{b} & \nabla_{z}^{b}\end{array}\right]^{T}$ and $\mathbf{w}_{a}^{b}$ are the accelerometer constant errors and random errors in $b$ frame. $\widetilde{\mathbf{f}}^{b}$ is the specific force measured by accelerometer. $\widetilde{\boldsymbol{\omega}}_{e n}^{n}$ is the angular velocity of the rotation of a navigation coordinates frame relative to the earth. $\widetilde{\boldsymbol{\omega}}_{i e}^{n}$ is the rotation projection of the earth. $\delta \boldsymbol{\omega}_{i n}^{n}, \delta \boldsymbol{\omega}_{i e}^{n}$, and $\delta \boldsymbol{\omega}_{e n}^{n}$ are the slow variations of $\widetilde{\boldsymbol{\omega}}_{i n}^{n}, \widetilde{\boldsymbol{\omega}}_{i e}^{n}$, and $\widetilde{\boldsymbol{\omega}}_{e n}^{n}$, respectively. $\mathbf{C}_{\omega}^{-1}$ is the inverse matrix of $\mathbf{C}_{\omega}$. With the large misalignment, $\mathbf{C}_{\omega}$ and $\mathbf{C}_{n}^{n^{\prime}}$ can be described as

$$
\begin{aligned}
\mathbf{C}_{n}^{n^{\prime}} & =\left[\begin{array}{ccc}
\cos \phi_{n} \cos \phi_{u}-\sin \phi_{n} \sin \phi_{e} \sin \phi_{u} & \cos \phi_{n} \sin \phi_{u}+\sin \phi_{n} \sin \phi_{e} \sin \phi_{u} & -\sin \phi_{n} \cos \phi_{e} \\
-\cos \phi_{e} \sin \phi_{u} & \cos \phi_{e} \cos \phi_{u} & \sin \phi_{e} \\
\sin \phi_{n} \cos \phi_{u}+\cos \phi_{n} \sin \phi_{e} \sin \phi_{u} & \sin \phi_{n} \sin \phi_{u}-\cos \phi_{n} \sin \phi_{e} \sin \phi_{u} & \cos \phi_{n} \cos \phi_{e}
\end{array}\right], \\
\mathbf{C}_{\omega} & =\left[\begin{array}{ccc}
\cos \phi_{n} & 0 & -\sin \phi_{n} \cos \phi_{e} \\
0 & 1 & \sin \phi_{e} \\
\sin \phi_{n} & 0 & \cos \phi_{n} \cos \phi_{e}
\end{array}\right] .
\end{aligned}
$$

The state vector is selected as

$$
\mathbf{x}=\left[\begin{array}{llllllllll}
\delta v_{e}^{n} & \delta v_{n}^{n} & \phi_{e} & \phi_{n} & \phi_{u} & \nabla_{x}^{b} & \nabla_{y}^{b} & \varepsilon_{x}^{b} & \varepsilon_{y}^{b} & \varepsilon_{z}^{b}
\end{array}\right] .
$$

On a moving base, the nonlinear state equation of SINS can be obtained as

$$
\begin{aligned}
\dot{\boldsymbol{\phi}}= & \mathbf{C}_{\omega}^{-1}\left[\left(I-\mathbf{C}_{n}^{n^{\prime}}\right) \widetilde{\boldsymbol{w}}_{i n}^{n}+\delta \boldsymbol{\omega}_{i n}^{n}-\mathbf{C}_{b}^{n^{\prime}} \boldsymbol{\varepsilon}^{b}\right]+\mathbf{w}_{g^{n}}^{n} \\
\delta \dot{\mathbf{v}}^{n}= & \left(\mathbf{I}-\mathbf{C}_{n^{\prime}}^{n}\right) \mathbf{f}^{n^{\prime}}-\left(2 \delta \boldsymbol{\omega}_{i e}^{n}+\delta \boldsymbol{\omega}_{e n}^{n}\right) \times \widetilde{\mathbf{v}}^{n} \\
& -\left(2 \widetilde{\boldsymbol{\omega}}_{i e}^{n}+\widetilde{\boldsymbol{w}}_{e n}^{n}\right) \times \delta \widetilde{\mathbf{v}}^{n}+\mathbf{C}_{n^{\prime}}^{n} \mathbf{C}_{b}^{n^{\prime}} \nabla^{b}+\mathbf{w}_{a}^{n},
\end{aligned}
$$


TABLE 1: Simulation conditions of swing base.

\begin{tabular}{lccc}
\hline & Roll & Pitch & Yaw \\
\hline Swaying amplitude $\left(^{\circ}\right)$ & 9 & 12 & 14 \\
Swaying oscillation cycles $(\mathrm{s})$ & 8 & 10 & 6 \\
\hline
\end{tabular}

TABLE 2: Simulation conditions of sensor precision.

\begin{tabular}{lccc}
\hline \multicolumn{2}{c}{ Gyroscope } & \multicolumn{2}{c}{ Accelerometer } \\
\hline Constant drift & $0.01^{\circ} / \mathrm{h}$ & Constant bias & $50 \mathrm{ug}$ \\
Random drift & $0.001^{\circ} / \sqrt{\mathrm{h}}$ & Random deviation & $50 \mathrm{ug}$ \\
\hline
\end{tabular}

$$
\begin{gathered}
\dot{\boldsymbol{\varepsilon}}^{b}=\mathbf{0}, \\
\dot{\boldsymbol{\nabla}}^{b}=\mathbf{0} .
\end{gathered}
$$

5.2. Establishment of the Measurement Equation. In this paper, we choose the east velocity and north velocity to establish the measurement equation. The velocity differential equation of SINS can be described as

$$
\mathbf{z}=\widetilde{\mathbf{v}}_{s}^{n}-\mathbf{C}_{n}^{n^{\prime}} \widetilde{\mathbf{v}}^{b}=\delta \mathbf{v}^{n}-\left[I-\left(C_{n}^{n^{\prime}}\right)^{T}\right] C_{b}^{n^{\prime}} \mathbf{v}^{b}+\mathbf{v},
$$

where $\widetilde{\mathbf{v}}_{s}^{n}$ and $\mathbf{v}^{b}$ are velocity calculated by SINS and the true value, respectively (only contains the east and north velocity components). $\widetilde{\mathbf{v}}^{b}$ includes the east and north external velocity information. $\mathbf{v}$ is the measurement noise.

If the external velocity information in $n$ frame $\widetilde{\mathbf{v}}^{n}$ is known, the measurement equation can be simplified as

$$
\mathbf{z}=\widetilde{\mathbf{v}}_{s}^{n}-\widetilde{\mathbf{v}}^{n}=\mathbf{H} \mathbf{x}+\mathbf{v},
$$

where $\widetilde{\mathbf{v}}^{n}$ is the external velocity vector. $\mathbf{H}$ is the measurement matrix and $\mathbf{H}=\left[\begin{array}{ll}\mathbf{I}_{2 \times 2} & \mathbf{0}_{2 \times 8}\end{array}\right]$.

\section{Experiments and Analysis}

6.1. Mathematical Simulation. In this part, the navigation system is placed on a simulated swing base and the initial misalignment angles are set to large values: $\Delta \theta=10^{\circ}, \Delta \gamma=$ $10^{\circ}$, and $\Delta \psi=15^{\circ}, 20^{\circ}, 35^{\circ}, 40^{\circ}$, respectively. The simulation conditions of swing base and sensors are in Tables 1 and 2 . Set system noise as zero-mean Gaussian white noise. Also assume that velocity of navigation system is $5 \mathrm{~m} / \mathrm{s}$ and the external information only includes the velocity in $b$ frame. The SRCKF, TUKF, and TUQKF-2 are used to solve this initial alignment problem. Simulation time is $700 \mathrm{~s}$. A comparison of these three methods is given in detail.

The simulation results are shown in Table 3 and the curve graphs of alignment errors with $\Delta \theta=10^{\circ}, \Delta \gamma=10^{\circ}$, and $\Delta \psi=40^{\circ}$ are shown in Figure 1.

Analyze the accuracy of initial alignment first. In Figure 1, when $\Delta \theta=10^{\circ}, \Delta \gamma=10^{\circ}$, and $\Delta \psi=40^{\circ}$, after $600 \mathrm{~s}$, the error curves of horizontal alignment of 3 methods are closed and errors are less than $0.01^{\circ}$. The azimuth alignment errors of TUQKF-2 and TUKF are less than $0.05^{\circ}$ and $0.1^{\circ}$, respectively. Their precisions are much better than SRCKF of which azimuth alignment error is more than $0.2^{\circ}$. So, in this simulation, the precision of TUQKF-2 is better than TUKF and much better than SRCKF.

In Table 3, the same results can be seen. For different azimuth misalignment angles, the average errors of horizontal alignment of 3 methods are all less than $0.008^{\circ}$ and the average error of azimuth alignment of TUQKF-2 is better than TUKF and SRCKF. So, the TUQKF-2 algorithm can maintain the high accuracy of horizontal alignment and remarkably improve the accuracy of azimuth alignment.

Next, analyze the convergence speed. In Figure 1, the convergence speed of TUQKF-2 and TUKF are almost the same. The horizontal alignment convergence time is less than $200 \mathrm{~s}$ and the azimuth alignment convergence time is less than $500 \mathrm{~s}$. Compared to these, the convergence speed of SRCKF is very slow.

Owing to the convergence speed of azimuth alignment which is faster than horizontal alignment, we calculated the standard deviation of azimuth estimation errors in different periods to quantitatively analyze the convergence speed of azimuth alignment. The concrete data are shown in Table 4.

From Table 4, it can be seen that, in most of the time, the standard deviation of azimuth estimation error of TUQKF-2 is a bit smaller than TUKF and much smaller than SRCKF. So, the convergence speed of the azimuth of TUQKF-2 is a bit faster than TUKF and much faster than SRCKF.

In a word, according to the mathematical simulation, the accuracy of horizontal alignment of 3 methods is almost same, less than $0.01^{\circ}$. The precision of azimuth alignment of TUQKF-2 is better than TUKF and SRCKF. The convergence speed, especially the convergence speed of azimuth alignment of TUQKF-2, is a little better than TUKF and much better than the SRCKF.

6.2. Turntable Experiment. To verify the effectiveness of the RTUQKF, turntable experiment is designed. In this turntable experiment, one SINS named FOSN is placed on a three-axis turntable. FOSN can provide gyroscope data and accelerometer data. The sensor accuracy of FOSN is given in Table 5. The precision of turntable is $\pm 0.0001^{\circ}$, so it can provide the true attitude angles as a reference. In the experiment, the FOSN is connected with the turntable; the turntable is controlled by the computer and makes a fixed swaying motion.

In this experiment, the data are collected from the real world, so the statistical properties of noise are uncertain and the velocity of SINS is zero, so (54) can be used as measurement equation.

Two tests with different initial attitudes, different swaying amplitudes, and different oscillation cycles are made. The SRCKF, TUQKF-2, and RTUQKF-2 are used to solve the above initial alignment problem, respectively. The tests conditions and results are as follows.

6.2.1. The First Turntable Test. In this test, 3 different kinds of initial attitude angles are selected as $\widetilde{\theta}=15^{\circ}, \widetilde{\gamma}=10^{\circ}$, and $\widetilde{\psi}=$ $35^{\circ}$ and $\widetilde{\theta}=15^{\circ}$, and $\widetilde{\gamma}=10^{\circ}, \widetilde{\psi}=30^{\circ}$, and $\widetilde{\theta}=10^{\circ}, \widetilde{\gamma}=5^{\circ}$, and $\widetilde{\psi}=30^{\circ}$. True initial attitude angles are $\theta=6.9443^{\circ}$, 
TABLE 3: Statistical properties of alignment errors in mathematical simulation.

\begin{tabular}{|c|c|c|c|c|c|c|c|}
\hline \multirow{2}{*}{ Algorithm } & \multirow{2}{*}{$\Delta \psi\left(\left(^{\circ}\right)\right.$} & \multicolumn{2}{|c|}{ Pitch error $\left({ }^{\circ}\right)$} & \multicolumn{2}{|c|}{ Roll error $\left(^{\circ}\right)$} & \multicolumn{2}{|c|}{ Yaw error $\left({ }^{\circ}\right)$} \\
\hline & & Mean & Standard deviation & Mean & Standard deviation & Mean & Standard deviation \\
\hline SRCKF & & 0.0023 & 0.00031 & -0.0017 & 0.00042 & 0.0945 & 0.0202 \\
\hline TUKF & 15 & 0.0078 & 0.00047 & -0.0026 & 0.0014 & 0.0594 & 0.0068 \\
\hline TUQKF & & 0.0035 & 0.00045 & -0.0025 & 0.00064 & 0.0130 & 0.0090 \\
\hline SRCKF & & 0.0021 & 0.00032 & -0.0011 & 0.00039 & 0.1264 & 0.0235 \\
\hline TUKF & 20 & 0.0080 & 0.00071 & -0.0041 & 0.0015 & 0.0538 & 0.0064 \\
\hline TUQKF & & 0.0034 & 0.00058 & -0.0033 & 0.00066 & 0.0188 & 0.0096 \\
\hline SRCKF & & 0.0033 & 0.00063 & 0.0016 & 0.0006 & 0.2019 & 0.0359 \\
\hline TUKF & 35 & 0.0075 & 0.00068 & -0.0040 & 0.0014 & -0.0713 & 0.0062 \\
\hline TUQKF & & 0.0043 & 0.00064 & -0.0037 & 0.00079 & -0.0090 & 0.0081 \\
\hline SRCKF & & 0.0026 & 0.00094 & 0.0035 & 0.0005 & 0.3270 & 0.0503 \\
\hline TUKF & 40 & 0.0073 & 0.00074 & -0.0043 & 0.0013 & -0.0726 & 0.0070 \\
\hline TUQKF & & 0.0044 & 0.00072 & -0.0042 & 0.00082 & -0.0138 & 0.0070 \\
\hline
\end{tabular}
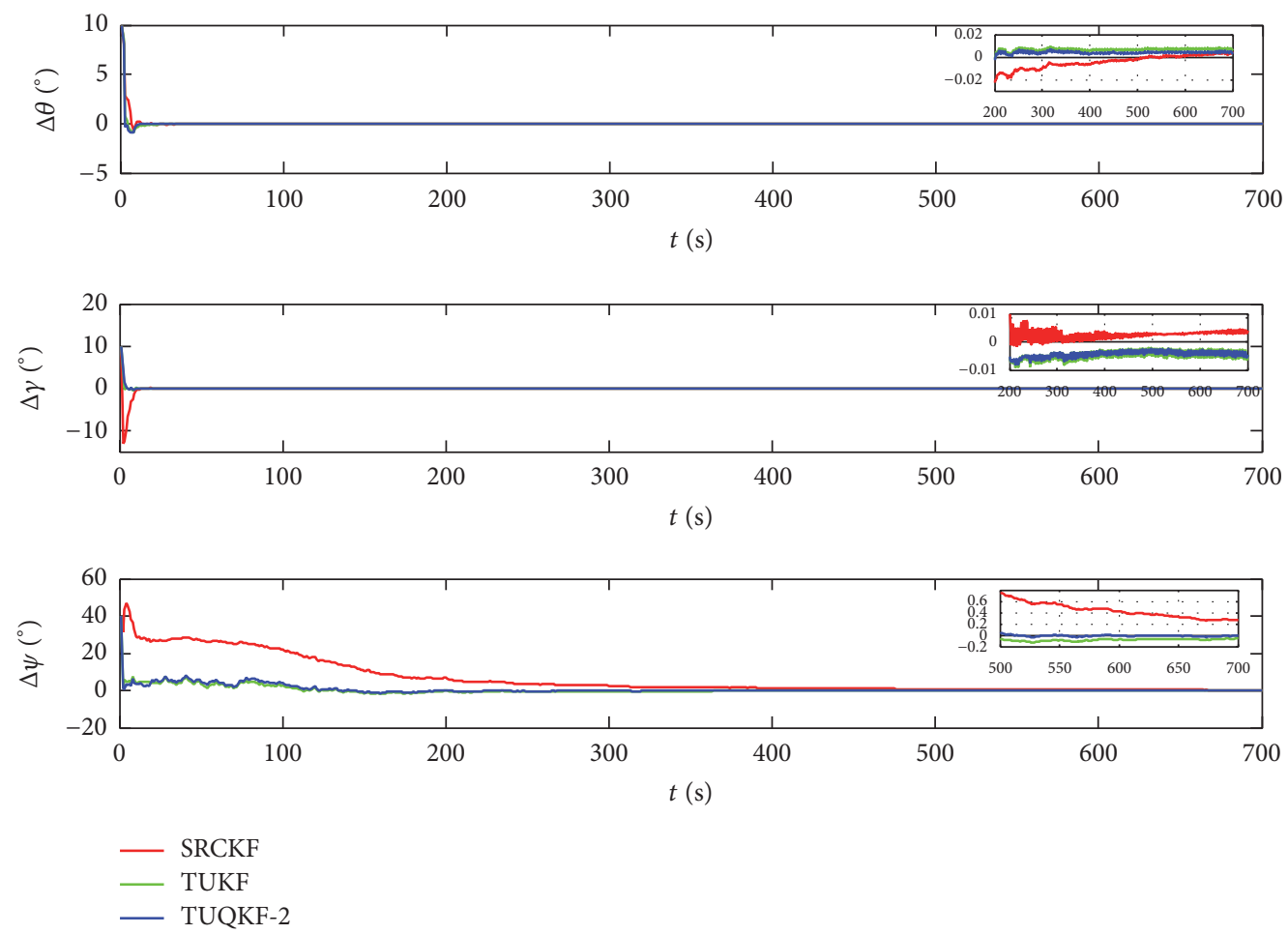

FIGURE 1: Alignment errors of mathematical simulation.

$\gamma=-0.5263^{\circ}$, and $\psi=-5.7903^{\circ}$, so initial misalignment angles are large. The swaying amplitudes of inner frame (roll), intermediate frame (pitch), and outer frame (yaw) are $10^{\circ}, 12^{\circ}$, and $6^{\circ}$, respectively. The corresponding oscillation frequencies are $0.2 \mathrm{~Hz}, 0.125 \mathrm{~Hz}$, and $0.15 \mathrm{~Hz}$, respectively. Simulation time is also $700 \mathrm{~s}$. The experiment results are shown in Table 6 and the curve graphs of alignment errors with $\widetilde{\theta}=15^{\circ}, \widetilde{\gamma}=10^{\circ}$, and $\widetilde{\psi}=35^{\circ}$ are shown in Figure 2 .

In Figure 2 , when $\widetilde{\theta}=15^{\circ}, \widetilde{\gamma}=10^{\circ}$, and $\widetilde{\psi}=35^{\circ}$, after $600 \mathrm{~s}$, the initial errors of pitch and roll angles of 3 methods are also closed and less than about $0.04^{\circ}$ and $0.05^{\circ}$. It is obviously that azimuth alignment error of RTUQKF-2 is better than TUQKF-2 and much better than SRCKF.

In Table 6, for different initial attitude angles, mean errors of horizontal alignment of 3 methods are all very small and in the same level. The average error of azimuth alignment of RTUQKF-2 is around $0.03^{\circ}$ and much less than the TUQKF2 and SRCKF if which the mean of azimuth alignment error is around $0.05^{\circ}$ and $0.5^{\circ}$, respectively. So, the RTUQKF2 algorithm can maintain the high accuracy of horizontal alignment and remarkably improve the accuracy of azimuth alignment when the misalignment angles are large and the 
TABLE 4: Standard deviation of azimuth estimation errors in mathematical simulation.

\begin{tabular}{|c|c|c|c|c|c|c|}
\hline \multirow{2}{*}{ Algorithm } & \multirow{2}{*}{$\Delta \psi\left({ }^{\circ}\right)$} & \multicolumn{5}{|c|}{ Standard deviation of azimuth estimation errors in different periods $\left(^{\circ}\right)$} \\
\hline & & $100 \sim 200 \mathrm{~s}$ & $200 \sim 300 \mathrm{~s}$ & $300 \sim 400 \mathrm{~s}$ & $400 \sim 500 \mathrm{~s}$ & $500 \sim 600 \mathrm{~s}$ \\
\hline SRCKF & & 1.4951 & 0.3025 & 0.0806 & 0.0473 & 0.0366 \\
\hline TUKF & 15 & 0.4951 & 0.2337 & 0.1326 & 0.0325 & 0.0152 \\
\hline TUQKF & & 0.9351 & 0.1279 & 0.0737 & 0.0122 & 0.0175 \\
\hline SRCKF & & 2.0072 & 0.3769 & 0.0893 & 0.0580 & 0.0378 \\
\hline TUKF & 20 & 0.7823 & 0.1854 & 0.1191 & 0.0268 & 0.0144 \\
\hline TUQKF & & 1.1878 & 0.1286 & 0.0712 & 0.0129 & 0.0183 \\
\hline SRCKF & & 3.6417 & 0.8143 & 0.2009 & 0.1136 & 0.0630 \\
\hline TUKF & 35 & 1.6296 & 0.1776 & 0.1107 & 0.0224 & 0.0142 \\
\hline TUQKF & & 1.1948 & 0.1486 & 0.0858 & 0.0143 & 0.0164 \\
\hline SRCKF & & 4.8857 & 1.0047 & 0.2880 & 0.1618 & 0.0878 \\
\hline TUKF & 40 & 1.0020 & 0.1902 & 0.1270 & 0.0286 & 0.0153 \\
\hline TUQKF & & 1.1915 & 0.1572 & 0.0978 & 0.0167 & 0.0149 \\
\hline
\end{tabular}
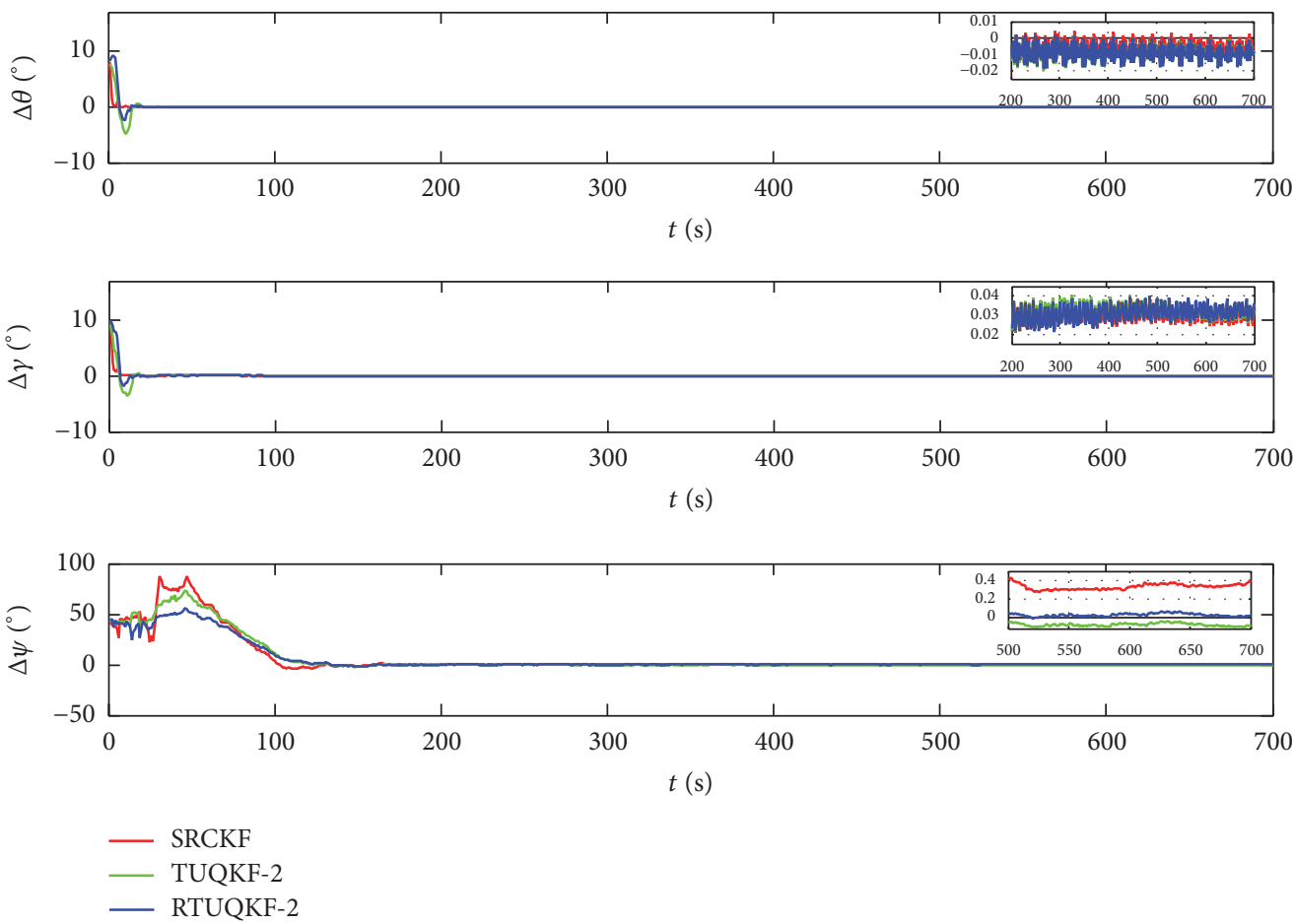

FIgURE 2: The alignment errors of first turntable test.

TABLE 5: The sensor accuracy of FOSN.

\begin{tabular}{lccc}
\hline \multicolumn{2}{c}{ Gyroscope } & \multicolumn{2}{c}{ Accelerometer } \\
\hline Constant drift & $0.006^{\circ} / \mathrm{h}$ & Constant bias & $50 \mathrm{ug}$ \\
Random drift & $0.006^{\circ} / \sqrt{\mathrm{h}}$ & Random deviation & $50 \mathrm{ug}$ \\
\hline
\end{tabular}

noise is uncertain. In addition, the precision of TUQKF declines when the actual noise is uncertain and the RTUQKF is more robust than the TUQKF.
Next, analyze the convergence speed. In Figure 2, convergence speed of 3 methods is almost the same; only the convergence speed of azimuth alignment of SRCKF is slightly slower than other two methods. We also calculated the standard deviation of azimuth estimation errors in different periods to quantitatively analyze the convergence rate of alignment. The concrete data are shown in Table 7.

From Table 7, the standard deviation of azimuth estimation errors of 3 methods are almost the same. It means that convergence speeds of 3 methods are all fast. However, when initial misalignment angles are very large, the standard 
TABLE 6: Statistical properties of alignment errors in first turntable test.

\begin{tabular}{|c|c|c|c|c|c|c|c|}
\hline \multirow{2}{*}{ Algorithm } & \multirow{2}{*}{$\tilde{\theta}, \tilde{\gamma}, \tilde{\psi}\left(^{\circ}\right)$} & \multicolumn{2}{|c|}{ Pitch error $\left(^{\circ}\right)$} & \multicolumn{2}{|c|}{ Roll error $\left(^{\circ}\right)$} & \multicolumn{2}{|c|}{ Yaw error $\left({ }^{\circ}\right)$} \\
\hline & & Mean & Standard deviation & Mean & Standard deviation & Mean & Standard deviation \\
\hline SRCKF & & -0.0100 & 0.0039 & -0.0322 & 0.0033 & 0.2472 & 0.0234 \\
\hline TUQKF & $10,5,30$ & -0.0101 & 0.0040 & -0.0353 & 0.0033 & 0.0427 & 0.0221 \\
\hline RTUQKF & & -0.0079 & 0.0039 & 0.0327 & 0.0032 & 0.0257 & 0.0156 \\
\hline SRCKF & & -0.0090 & 0.0039 & 0.0335 & 0.0032 & 0.2603 & 0.0238 \\
\hline TUQKF & $15,10,30$ & -0.0101 & 0.0039 & 0.0335 & 0.0033 & -0.0560 & 0.0201 \\
\hline RTUQKF & & -0.0101 & 0.0038 & 0.0318 & 0.0033 & 0.0211 & 0.0162 \\
\hline SRCKF & & -0.0059 & 0.0038 & 0.0295 & 0.0032 & 0.3515 & 0.0150 \\
\hline TUQKF & $15,10,35$ & -0.0093 & 0.0038 & 0.0311 & 0.0032 & -0.0741 & 0.0182 \\
\hline RTUQKF & & -0.0096 & 0.0039 & 0.0318 & 0.0033 & 0.0344 & 0.0165 \\
\hline
\end{tabular}

TABLE 7: Standard deviation of azimuth estimation errors in first turntable test.

\begin{tabular}{|c|c|c|c|c|c|c|}
\hline \multirow{2}{*}{ Algorithm } & \multirow{2}{*}{$\tilde{\theta}, \tilde{\gamma}, \widetilde{\psi}\left({ }^{\circ}\right)$} & \multicolumn{5}{|c|}{ Standard deviation of azimuth estimation errors in different periods $\left(^{\circ}\right)$} \\
\hline & & $100 \sim 200 \mathrm{~s}$ & $200 \sim 300 \mathrm{~s}$ & $300 \sim 400 \mathrm{~s}$ & $400 \sim 500 \mathrm{~s}$ & $500 \sim 600 \mathrm{~s}$ \\
\hline SRCKF & \multirow{3}{*}{$10,5,30$} & 1.4738 & 0.1286 & 0.0428 & 0.0225 & 0.0251 \\
\hline TUQKF & & 2.7423 & 0.1286 & 0.0528 & 0.0332 & 0.0196 \\
\hline RTUQKF & & 1.3892 & 0.1481 & 0.0492 & 0.0190 & 0.0145 \\
\hline SRCKF & \multirow{3}{*}{$15,10,30$} & 2.7479 & 0.1361 & 0.0339 & 0.0231 & 0.0299 \\
\hline TUQKF & & 2.3790 & 0.1729 & 0.0452 & 0.0283 & 0.0157 \\
\hline RTUQKF & & 1.5035 & 0.1410 & 0.0448 & 0.0186 & 0.0140 \\
\hline SRCKF & \multirow{3}{*}{$15,10,35$} & 1.5865 & 0.1704 & 0.0520 & 0.0200 & 0.0320 \\
\hline TUQKF & & 2.3692 & 0.1637 & 0.0449 & 0.0214 & 0.0139 \\
\hline RTUQKF & & 2.3244 & 0.1371 & 0.0453 & 0.0187 & 0.0137 \\
\hline
\end{tabular}

TABLE 8: Statistical properties of the alignment errors in second turntable test.

\begin{tabular}{|c|c|c|c|c|c|c|c|}
\hline \multirow{2}{*}{ Algorithm } & \multirow{2}{*}{$\Delta \widetilde{\theta}, \Delta \widetilde{\gamma}, \Delta \widetilde{\psi}\left({ }^{\circ}\right)$} & \multicolumn{2}{|c|}{ Pitch error $\left({ }^{\circ}\right)$} & \multicolumn{2}{|c|}{ Roll error $\left({ }^{\circ}\right)$} & \multicolumn{2}{|c|}{ Yaw error $\left(^{\circ}\right)$} \\
\hline & & Mean & Standard deviation & Mean & Standard deviation & Mean & Standard deviation \\
\hline SRCKF & & -0.0056 & 0.0045 & -0.0216 & 0.0028 & 0.2789 & 0.0098 \\
\hline TUQKF & $5,5,25$ & -0.0074 & 0.0046 & -0.0209 & 0.0027 & 0.0411 & 0.0051 \\
\hline RTUQKF & & -0.0069 & 0.0046 & -0.0207 & 0.0027 & 0.0141 & 0.0050 \\
\hline SRCKF & & -0.0042 & 0.0044 & -0.0258 & 0.0028 & 0.4730 & 0.0310 \\
\hline TUQKF & $5,5,40$ & -0.0065 & 0.0045 & -0.0235 & 0.0028 & 0.0859 & 0.0098 \\
\hline RTUQKF & & -0.0058 & 0.0045 & -0.0218 & 0.0027 & 0.0361 & 0.0042 \\
\hline SRCKF & & -0.0049 & 0.0045 & -0.0236 & 0.0028 & 0.4346 & 0.0189 \\
\hline TUQKF & $10,10,35$ & -0.0061 & 0.0045 & -0.0235 & 0.0027 & 0.0836 & 0.0117 \\
\hline RTUQKF & & -0.0068 & 0.0045 & -0.0217 & 0.0027 & 0.0362 & 0.0042 \\
\hline
\end{tabular}

deviation of azimuth misalignment angles estimation errors of RTUQKF-2 is the smallest. So, the convergence speed of RTUQKF-2 is better than other two methods with large misalignment angles and uncertain noise.

6.2.2. The Second Turntable Test. In this test, initial misalignment angles are set to large values: $\Delta \theta=5^{\circ}, \Delta \gamma=5^{\circ}$, and $\Delta \psi=25^{\circ}$ and $\Delta \theta=5^{\circ}, \Delta \gamma=5^{\circ}$, and $\Delta \psi=40^{\circ}$, and $\Delta \theta=10^{\circ}, \Delta \gamma=5^{\circ}$, and $\Delta \psi=40^{\circ}$. The swaying amplitudes of inner frame (roll), intermediate frame (pitch), and outer frame (yaw) are $12^{\circ}, 8^{\circ}$, and $6^{\circ}$, respectively. The corresponding oscillation frequencies are $0.2 \mathrm{~Hz}, 0.125 \mathrm{~Hz}$, and $0.15 \mathrm{~Hz}$, respectively. The corresponding rocking center is $6^{\circ}, 8^{\circ}$, and $75^{\circ}$, respectively. Simulation time is $800 \mathrm{~s}$. The experiment results are shown in Table 8 and the curve graphs of the alignment errors with $\Delta \theta=10^{\circ}, \Delta \gamma=5^{\circ}$, and $\Delta \psi=40^{\circ}$ are shown in Figure 3. The standard deviations of azimuth estimation errors in different periods are shown in Table 9.

From Figure 3 and Table 8, the precisions of horizontal alignment of 3 methods are high and in the same level. The precision of azimuth alignment of the SRCKF is the lowest of the three methods. From Table 9, the convergence speed of TUQKF-2 is slightly better than other two filters.

It can be found that the results of two turntable tests are equally. In a word, according to the turntable experiment, when the noise is uncertain, precision of horizontal 

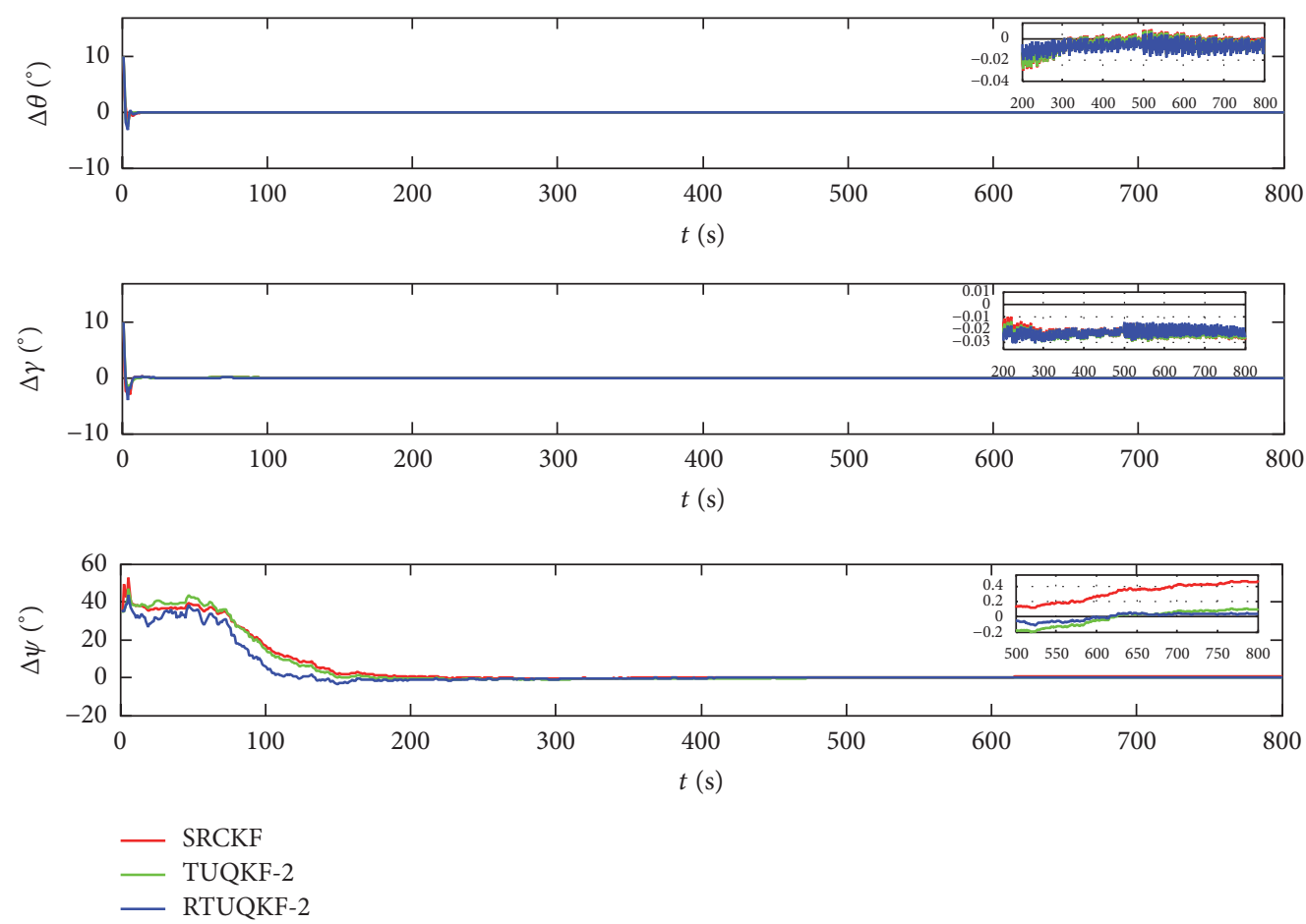

FIGURE 3: The alignment errors of second turntable test.

TABLE 9: Standard deviation of azimuth estimation errors in second turntable test.

\begin{tabular}{|c|c|c|c|c|c|c|c|}
\hline \multirow{2}{*}{ Algorithm } & \multirow{2}{*}{$\Delta \widetilde{\theta}, \Delta \widetilde{\gamma}, \Delta \widetilde{\psi}\left(^{\circ}\right)$} & \multicolumn{6}{|c|}{ Standard deviation of azimuth estimation errors in different periods $\left(^{\circ}\right)$} \\
\hline & & $100 \sim 200 \mathrm{~s}$ & $200 \sim 300 \mathrm{~s}$ & $300 \sim 400 \mathrm{~s}$ & $400 \sim 500 \mathrm{~s}$ & $500 \sim 600 \mathrm{~s}$ & $600 \sim 700 \mathrm{~s}$ \\
\hline SRCKF & \multirow{3}{*}{$5,5,25$} & 3.6450 & 0.2849 & 0.1276 & 0.0532 & 0.0316 & 0.0272 \\
\hline TUQKF & & 2.1568 & 0.2037 & 0.1623 & 0.0564 & 0.0264 & 0.0208 \\
\hline RTUQKF & & 2.1003 & 0.1842 & 0.1400 & 0.0512 & 0.0231 & 0.0191 \\
\hline SRCKF & \multirow{3}{*}{$5,5,40$} & 6.2382 & 0.5436 & 0.0952 & 0.0681 & 0.0480 & 0.0513 \\
\hline TUQKF & & 3.5482 & 0.1717 & 0.1686 & 0.0709 & 0.0333 & 0.0288 \\
\hline RTUQKF & & 2.2171 & 0.1970 & 0.1509 & 0.0600 & 0.0219 & 0.0180 \\
\hline SRCKF & \multirow{3}{*}{$10,10,35$} & 4.5488 & 0.3277 & 0.1279 & 0.0666 & 0.0403 & 0.0384 \\
\hline TUQKF & & 4.3622 & 0.2068 & 0.1619 & 0.0748 & 0.0414 & 0.0323 \\
\hline RTUQKF & & 1.6323 & 0.2280 & 0.1684 & 0.0612 & 0.0241 & 0.0191 \\
\hline
\end{tabular}

alignment of 3 methods is all high and in the same level. The RTUQKF can effectively increase the precision of azimuth alignment. The convergence speed of azimuth alignment of RTUQKF is better than the other algorithms when the misalignment angles are sufficiently large. It can be seen that the RTUQKF is more robust than the other two algorithms with the uncertain noise.

6.3. Vehicle Experiment. In the last, to further verify the effectiveness of RTUQKF, a vehicle experiment is conducted with the FOSN and PHINS. In this experiment, IMU data is given by FOSN. PHINS is a high precision SINS and it can be integrated with the FlexPark 6 from NovAtel as a SINS/GNSS integrated navigation system. The navigation parameters from PHINS are used as reference.

In the vehicle experiment, from start time to about 30 seconds, the vehicle is at the starting state and its velocity is zero. From about 30 seconds to about 780 seconds, the vehicle is in the moving state. After about 780 seconds, the vehicle stops moving. Alignment time is $800 \mathrm{~s}$. Owing to the external vehicle reference in $n$ frame which can be given, (54) can be used as measurement equation. In this experiment, the data are also collected from the real world, so the statistical properties of noise are uncertain.

Select two different kinds of misalignment angles for navigation system: $\Delta \theta=10^{\circ}, \Delta \gamma=10^{\circ}$, and $\Delta \psi=40^{\circ}$ and $\Delta \theta=5^{\circ}, \Delta \gamma=5^{\circ}$, and $\Delta \psi=30^{\circ}$. The SRCKF, TUQKF-2, and RTUQKF-2 are used to solve the above initial alignment problem, respectively. The vehicle experiment results are shown in Table 10 and the curve graphs of alignment errors with $\Delta \theta=10^{\circ}, \Delta \gamma=10^{\circ}$, and $\Delta \psi=40^{\circ}$ are shown in Figure 4.

In Figure 4 , when $\Delta \theta=10^{\circ}, \Delta \gamma=10^{\circ}$, and $\Delta \psi=40^{\circ}$, after $700 \mathrm{~s}$, the error curves of horizontal alignment of 3 methods are closed. It can also be seen obviously that error of azimuth 
TABLE 10: Statistical properties of the alignment errors in vehicle experiment.

\begin{tabular}{|c|c|c|c|c|c|c|c|}
\hline \multirow{2}{*}{ Algorithm } & \multirow{2}{*}{$\Delta \widetilde{\theta}, \Delta \widetilde{\gamma}, \Delta \widetilde{\psi}\left(\left(^{\circ}\right)\right.$} & \multicolumn{2}{|c|}{ Pitch error $\left({ }^{\circ}\right)$} & \multicolumn{2}{|c|}{ Roll error $\left({ }^{\circ}\right)$} & \multicolumn{2}{|c|}{ Yaw error $\left({ }^{\circ}\right)$} \\
\hline & & Mean & Standard deviation & Mean & Standard deviation & Mean & Standard deviation \\
\hline SRCKF & & 0.0102 & 0.0035 & -0.0134 & 0.0061 & -0.3330 & 0.0118 \\
\hline TUQKF & $5,5,30$ & 0.0072 & 0.0033 & -0.0123 & 0.0051 & -0.2595 & 0.0116 \\
\hline RTUQKF & & 0.0073 & 0.0031 & -0.0120 & 0.0050 & -0.2258 & 0.0118 \\
\hline SRCKF & & 0.0144 & 0.0042 & -0.0149 & 0.0074 & -0.4795 & 0.0192 \\
\hline TUQKF & $10,10,40$ & 0.0086 & 0.0039 & -0.0145 & 0.0059 & -0.3736 & 0.0148 \\
\hline RTUQKF & & 0.0089 & 0.0034 & -0.0133 & 0.0057 & -0.2585 & 0.0131 \\
\hline
\end{tabular}

TABLE 11: Standard deviation of azimuth estimation errors in vehicle experiment.

\begin{tabular}{|c|c|c|c|c|c|c|c|}
\hline \multirow{2}{*}{ Algorithm } & \multirow{2}{*}{$\Delta \widetilde{\theta}, \Delta \tilde{\gamma}, \Delta \widetilde{\psi}\left(^{\circ}\right)$} & \multicolumn{6}{|c|}{ Standard deviation of azimuth estimation errors in different periods $\left(^{\circ}\right)$} \\
\hline & & $100 \sim 200 \mathrm{~s}$ & $200 \sim 300 \mathrm{~s}$ & $300 \sim 400 \mathrm{~s}$ & $400 \sim 500 \mathrm{~s}$ & $500 \sim 600 \mathrm{~s}$ & $600 \sim 700 \mathrm{~s}$ \\
\hline SRCKF & \multirow{3}{*}{$5,5,30$} & 0.6754 & 0.0514 & 0.0846 & 0.0273 & 0.0291 & 0.0230 \\
\hline TUQKF & & 0.1251 & 0.0399 & 0.0363 & 0.0203 & 0.0230 & 0.0118 \\
\hline RTUQKF & & 0.1176 & 0.0361 & 0.0360 & 0.0207 & 0.0232 & 0.0119 \\
\hline SRCKF & \multirow{3}{*}{$10,10,40$} & 1.6544 & 0.0676 & 0.1785 & 0.0367 & 0.0533 & 0.0620 \\
\hline TUQKF & & 0.3962 & 0.0554 & 0.0350 & 0.0322 & 0.0323 & 0.0227 \\
\hline RTUQKF & & 0.3592 & 0.0594 & 0.0357 & 0.0153 & 0.0309 & 0.0154 \\
\hline
\end{tabular}

alignment of RTUQKF-2 is better than TUQKF-2 and much better than SRCKF. The data from Table 10 show that, for two different misalignment angles, average errors of horizontal alignment of 3 methods are very small and the average error of azimuth alignment of RTUQKF-2 is around $0.25^{\circ}$ and much less than the TUQKF-2 and SRCKF. This means that, for SINS in-motion alignment, RTUQKF-2 can maintain the high horizontal alignment accuracy and remarkably improve the accuracy of azimuth alignment when the misalignment angles are large and noise is uncertain.

The convergence speed is also quantitatively analyzed by the standard deviation of azimuth estimation errors in different periods in Table 11. From Figure 4 and Table 11, it can be seen that the standard deviation of azimuth misalignment angles estimation errors of SRCKF is larger than the other two methods. In most of the time, the standard deviation of azimuth misalignment angles estimation errors of RTUQKF2 slightly is smaller than TUQKF-2. It means that convergence speed of azimuth alignment of RTUQKF is slightly faster than TUQKF and faster than SRCKF.

The results of vehicle experiment are similar to turntable experiment. In conclusion, when misalignment angles are large and the noise is uncertain, the precision of the horizontal alignment of 3 methods is high. The superiority of RTUQKF embodies several aspects that this method can effectively increase the precision and convergence speed of azimuth alignment and this method improves the robustness of the algorithm when the noise is uncertain.

\section{Conclusions}

The SINS initial alignment problem is discussed in this paper. To solve the initial alignment problem under large misalignment angles, TUQKF algorithm is proposed. The TUQKF can maintain the positive definiteness of the weight and the covariance matrix in the filtering process and solve the nonlocal sampling problem for high-dimensional problem coupled with strong nonlinear model. In this paper, to solve the problem of uncertain noise in initial alignment, the RTUQKF algorithm is proposed. The RTUQKF is the combination of $H_{\infty}$ filtering and TUQKF. This method maintains the high accuracy of filtering and further improves the robustness of filtering. The performance of TUQKF is compared with other filters in mathematical simulation. The results indicate that, compared to traditional filters such as SRCKF and TUKF, TUQKF can increase precision and convergence speed of initial alignment. By means of the turntable experiment and vehicle experiment, the performance of RTUQKF is analyzed and compared with other filters. The results show that when the noise is uncertain, RTUQKF can further increase the precision and convergence speed of azimuth alignment and it can also effectively improve the robustness of filter. Although the computational cost of TUQKF and RTUQKF is slightly higher than SRCKF and TUKF, their sigma points have a linear relationship to state dimension and the order of quadrature rule. These two algorithms will not surfer from the curse of dimensionality problem.

\section{Competing Interests}

The authors declare that they have no competing interests.

\section{Acknowledgments}

The research was supported in part by the National Natural Science Foundation of China (Grants nos. 51175082, 61473085, and 51375088), Foundation of Key Laboratory of Micro-Inertial Instrument and Advanced Navigation 

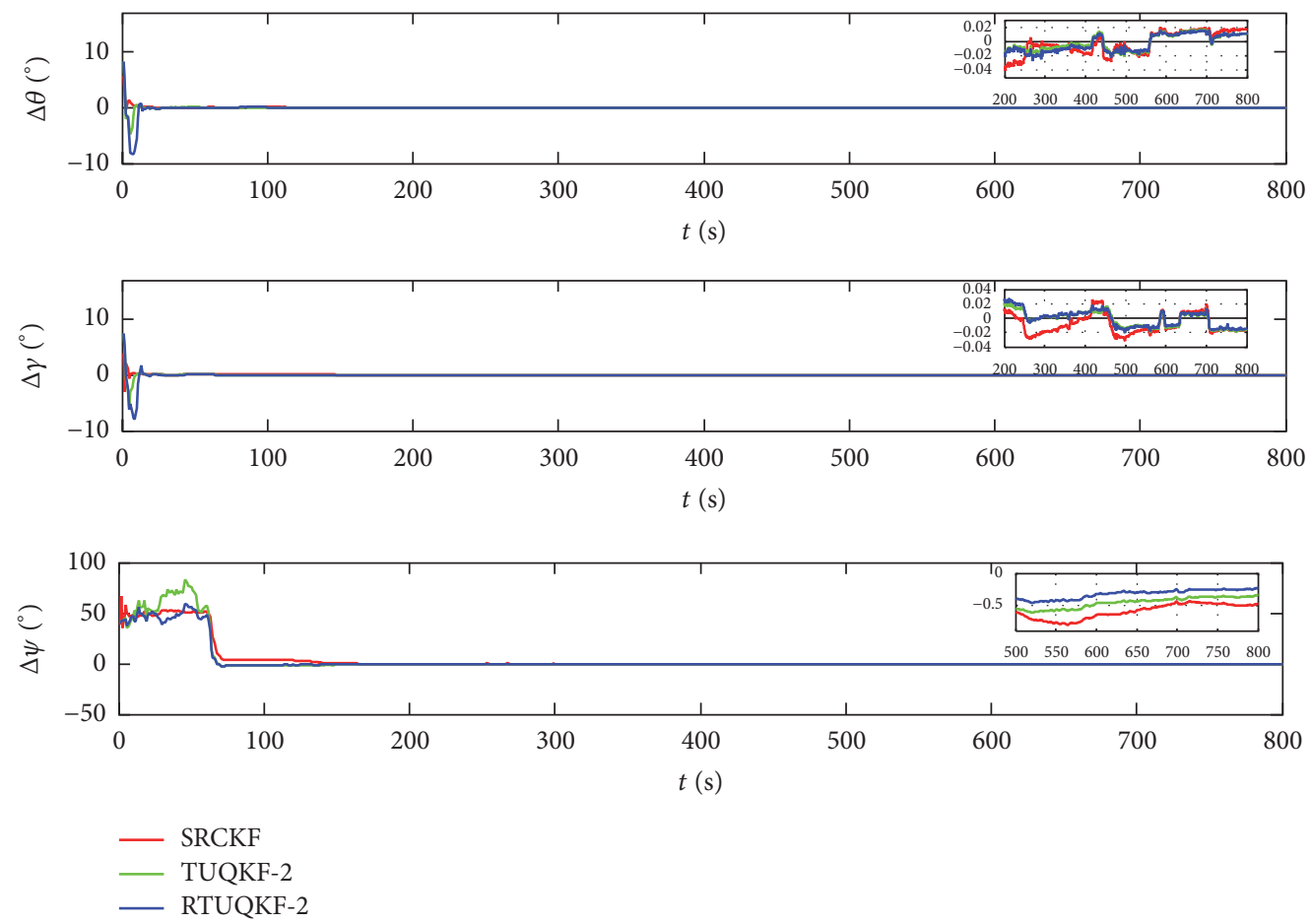

FIgURE 4: The alignment errors of vehicle experiment.

Technology of Ministry of Education of China (201403), Fundamental Research Funds for the Central Universities (2242015R30031), and Key Laboratory Fund of Ministry of Public Security based on a data structure (2015DSJSYS002).

\section{References}

[1] X. Liu, Y. Zhao, Z. Liu, and L. Wang, "A novel self-alignment method for SINS based on parameter recognition and dualvelocity vectors," Proceedings of the Institution of Mechanical Engineers, Part G: Journal of Aerospace Engineering, vol. 229, no. 12, pp. 2151-2162, 2015.

[2] D. Titterton and J. Weston, Strapdown Inertial Navigation Technology, IET, Stevenage, UK, 2nd edition, 2004.

[3] X. Liu, X. Xu, Y. Liu, and L. Wang, "A method for SINS alignment with large initial misalignment angles based on Kalman filter with parameters resetting," Mathematical Problems in Engineering, vol. 2014, Article ID 346291, 10 pages, 2014.

[4] J. Sun, X.-S. Xu, Y.-T. Liu, T. Zhang, and Y. Li, "Initial alignment of large azimuth misalignment angles in SINS based on adaptive UPF," Sensors, vol. 15, no. 9, pp. 21807-21823, 2015.

[5] T. Zhang and X.-S. Xu, "Research on SINS initial alignment and the methods for the parameter $\gamma$ selection based on $\mathrm{H}_{\infty}$ filtering technique," Journal of Sichuan University (Engineering Science Edition), vol. 41, no. 4, pp. 240-245, 2009.

[6] Y. Gongmin, Y. Weisheng, and X. Demin, "A SINS nonlinear error model reflecting better characteristics of SINS errors," Journal of Northwestern Polytechnical University, vol. 4, pp. 511516, 2009.

[7] S. P. Dmitriyev, O. A. Stepanov, and S. V. Shepel, "Nonlinear filtering methods application in INS alignment," IEEE Transactions on Aerospace and Electronic Systems, vol. 33, no. 1, pp. 260-272, 1997.
[8] S. J. Julier and J. K. Uhlmann, "Unscented filtering and nonlinear estimation," Proceedings of the IEEE, vol. 92, no. 3, pp. 401422, 2004.

[9] S. Julier, J. Uhlmann, and H. F. Durrant-Whyte, "A new method for the nonlinear transformation of means and covariances in filters and estimators," IEEE Transactions on Automatic Control, vol. 45, no. 3, pp. 477-482, 2000.

[10] I. Arasaratnam and S. Haykin, "Cubature kalman filters," Institute of Electrical and Electronics Engineers. Transactions on Automatic Control, vol. 54, no. 6, pp. 1254-1269, 2009.

[11] G.-M. Yan, W.-S. Yan, and D.-M. Xu, "Application of simplified UKF in SINS initial alignment for large misalignment angles," Journal of Chinese Inertial Technology, vol. 16, pp. 253-264, 2008.

[12] F. Sun and L. Tang, "Initial alignment of large azimuth misalignment angle in SINS based on CKF," Chinese Journal of Scientific Instrument, vol. 33, no. 2, pp. 327-333, 2012.

[13] S. J. Julier, "The scaled unscented transformation," in Proceedings of the IEEE American Control Conference, pp. 4555-4559, Anchorage, Alaska, USA, May 2002.

[14] L. Chang, B. Hu, A. Li, and F. Qin, "Transformed unscented Kalman filter," IEEE Transactions on Automatic Control, vol. 58, no. 1, pp. 252-257, 2013.

[15] B. Jia and M. Xin, "Sparse-grid quadrature $H_{\infty}$ filter for discrete-time systems with uncertain noise statistics," IEEE Transactions on Aerospace and Electronic Systems, vol. 49, no. 3, pp. 1626-1636, 2013.

[16] K. P. Chandra, D.-W. Gu, and I. Postlethwaite, "A cubature Ho filter and its square-root version," International Journal of Control, vol. 87, no. 4, pp. 764-776, 2014.

[17] G. A. Einicke and L. B. White, "Robust extended Kalman filtering," IEEE Transactions on Signal Processing, vol. 47, no. 9, pp. 2596-2599, 1999. 
[18] B. Zhou and X. Cheng, "Robust UKF algorithm in SINS initial alignment," Journal of Southeast University, vol. 27, no. 1, pp. 5660, 2011.

[19] K. Ito and K. Xiong, "Gaussian filters for nonlinear filtering problems," IEEE Transactions on Automatic Control, vol. 45, no. 5, pp. 910-927, 2000.

[20] I. Arasaratnam, S. Haykin, and R. J. Elliott, "Discrete-Time nonlinear filtering algorithms using Gauss-Hermite quadrature," Proceedings of the IEEE, vol. 95, no. 5, pp. 953-977, 2007.

[21] B. Jia, M. Xin, and Y. Cheng, "Sparse-grid quadrature nonlinear filtering," Automatica. A Journal of IFAC, the International Federation of Automatic Control, vol. 48, no. 2, pp. 327-341, 2012.

[22] S. Bhaumik and M. Swati, "Cubature quadrature kalman filter," IET Signal Processing, vol. 7, pp. 533-541, 2013.

[23] H. Chen, X. Cheng, C. Dai, and F. Liu, "Robust stability analysis of Ho-SGQKF and its application to transfer alignment," Signal Processing, vol. 117, pp. 310-321, 2015.

[24] A. H. Sayed, "A framework for state-space estimation with uncertain models," IEEE Transactions on Automatic Control, vol. 46, no. 7, pp. 998-1013, 2001.

[25] B. Hassibi, A. H. Sayed, and T. Kailath, Indefinite-Quadratic Estimation and Control: A Unified Approach to $\mathrm{H} 2$ and $\mathrm{H} \infty$ Theories, SIAM Studies in Applied Mathematics, Society for Industrial and Applied Mathematics (SIAM), Philadelphia, PA, Philadelphia, Pa, USA, 1999.

[26] D.-J. Lee, "Nonlinear estimation and multiple sensor fusion using unscented information filtering," IEEE Signal Processing Letters, vol. 15, pp. 861-864, 2008.

[27] W. Li and Y. Jia, "H-infinity filtering for a class of nonlinear discrete-time systems based on unscented transform," Signal Processing, vol. 90, no. 12, pp. 3301-3307, 2010. 


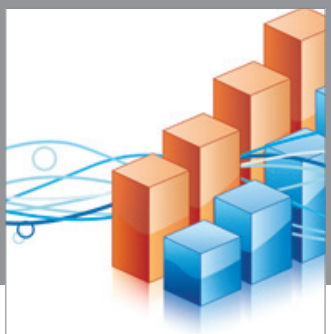

Advances in

Operations Research

vatem alat4

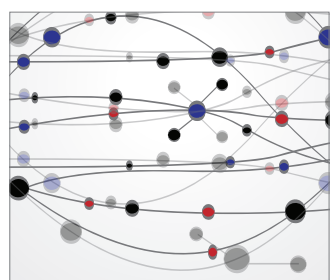

\section{The Scientific} World Journal
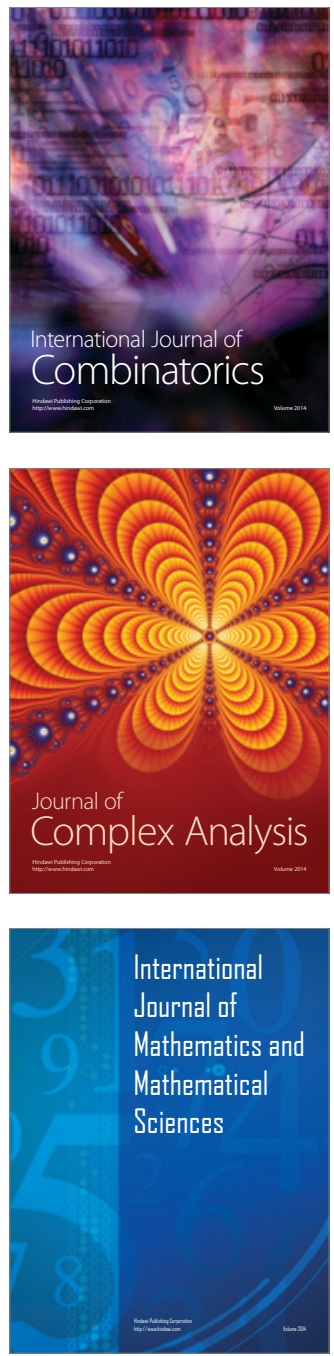
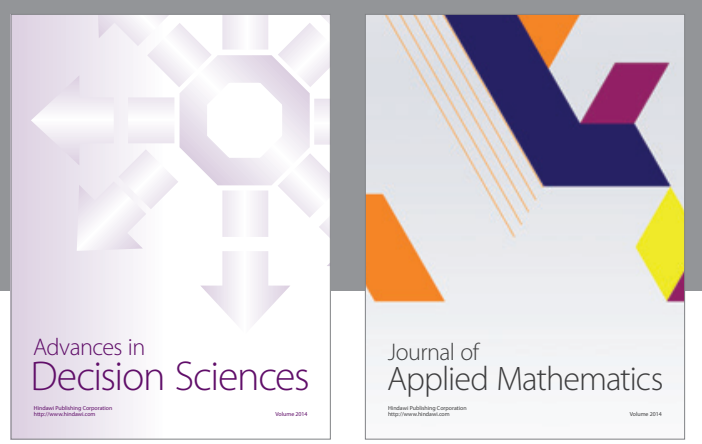

Algebra

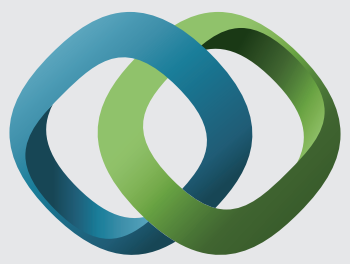

\section{Hindawi}

Submit your manuscripts at

https://www.hindawi.com
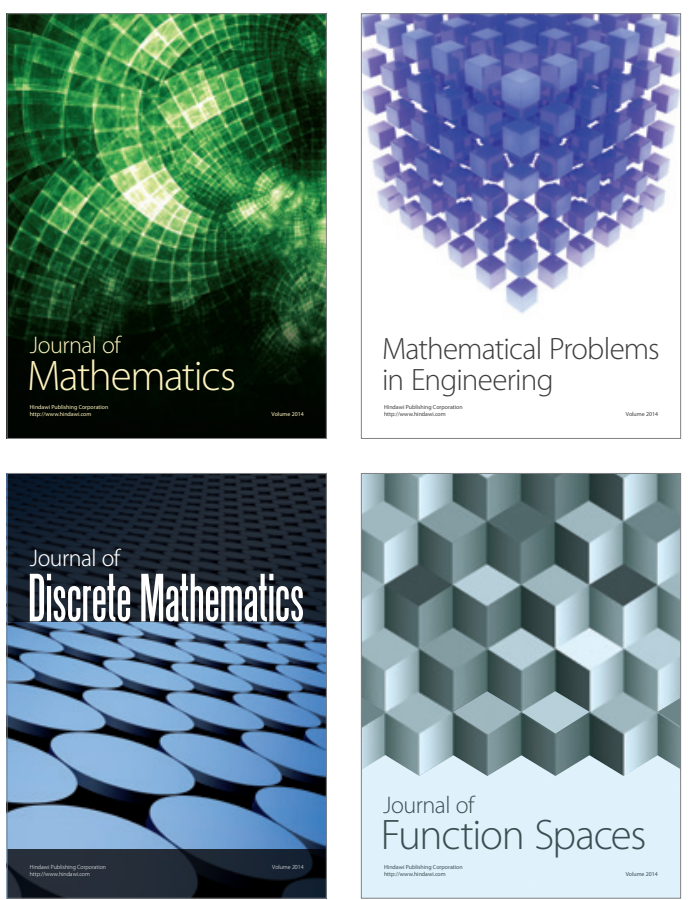

Mathematical Problems in Engineering
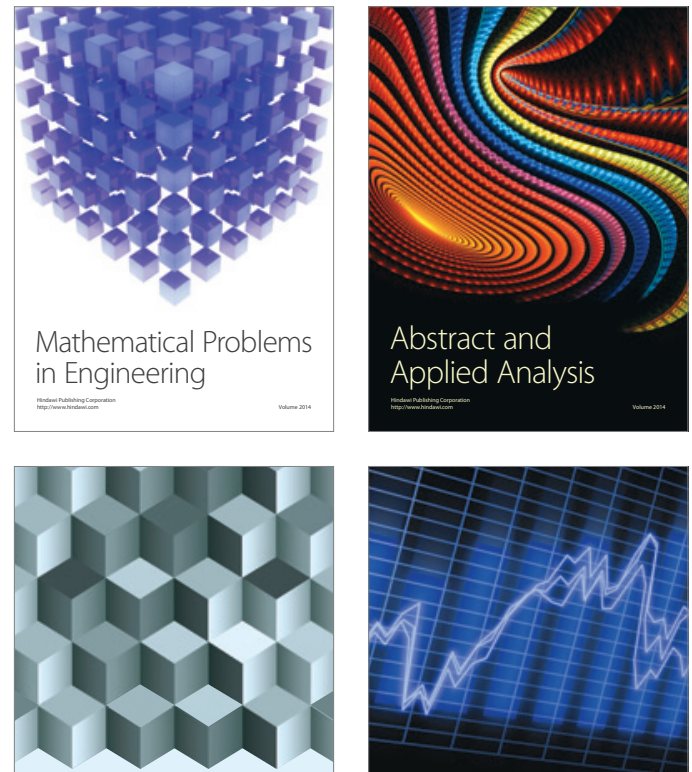

Journal of

Function Spaces

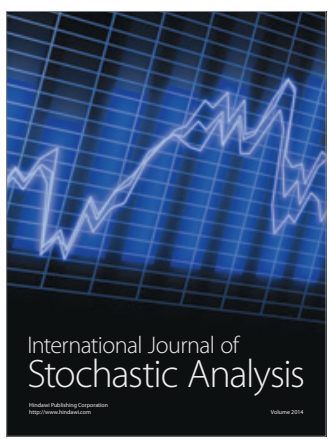

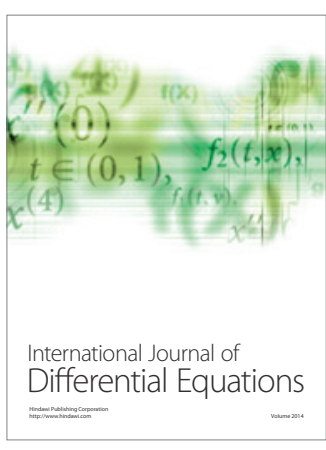
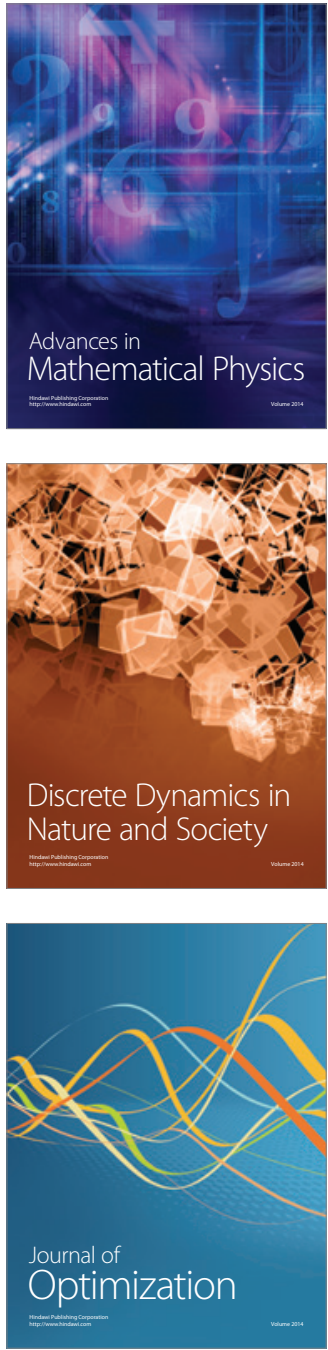